\title{
A Methodology to Find the Elementary Landscape Decomposition of Combinatorial Optimization Problems
}

\section{Francisco Chicano}

chicano@lcc.uma.es

Departamento de Lenguajes y Ciencias de la Computación, Universidad de Málaga, Spain

\author{
L. Darrell Whitley \\ whitley@cs.colostate.edu \\ Department of Computer Science, Colorado State University, USA
}

Enrique Alba

Departamento de Lenguajes y Ciencias de la Computación,

eat@lcc.uma.es

Universidad de Málaga, Spain

\begin{abstract}
A small number of combinatorial optimization problems have search spaces that correspond to elementary landscapes, where the objective function $f$ is an eigenfunction of the Laplacian that describes the neighborhood structure of the search space. Many problems are not elementary; however, the objective function of a combinatorial optimization problem can always be expressed as a superposition of multiple elementary landscapes if the underlying neighborhood used is symmetric. This paper presents theoretical results that provide the foundation for algebraic methods that can be used to decompose the objective function of an arbitrary combinatorial optimization problem into a sum of subfunctions, where each subfunction is an elementary landscape. Many steps of this process can be automated, and indeed a software tool could be developed that assists the researcher in finding a landscape decomposition. This methodology is then used to show that the subset sum problem is a superposition of two elementary landscapes, and to show that the quadratic assignment problem is a superposition of three elementary landscapes.
\end{abstract}

\section{Keywords}

Elementary landscape, fitness landscape, combinatorial optimization, decomposition methodology.

\section{Introduction}

Landscape analysis focuses on the analysis of the structure of the search space that is induced by the combined influences of the objective function of the optimization problem and the choice neighborhood operator (Stadler, 1995). This theory has applications not only in evolutionary computation (Whitley et al., 2008) but also in chemistry (Stadler, 1996), biology (Weinberger, 1990), and physics (García-Pelayo and Stadler, 1997).

A landscape for a combinatorial optimization problem is a triple $(X, N, f)$, where $f: X \mapsto \mathbb{R}$ defines the objective function and the neighborhood operator function $N(x)$ generates the set of points reachable from $x \in X$ in a single application of the neighborhood 
operator. If $y \in N(x)$, then $y$ is a neighbor of $x$. Elementary landscapes are a type of landscape which are of particular interest due to their special properties (Whitley et al., 2008). They are characterized by a wave equation:

$$
\underset{y \in N(x)}{\operatorname{avg}\{f(y)\}}=f(x)+\frac{k}{d}(\bar{f}-f(x)),
$$

where $d$ is the size of the neighborhood, $|N(x)|$, which we assume is the same for all the solutions in the search space; $\bar{f}$ is the average solution evaluation over the entire search space; and $k$ is a characteristic constant. The wave equation makes it possible to compute the average value of the fitness function $f$ evaluated over all of the neighbors of $x$ using only the value $f(x)$; we denote this average by using avg $\{f(y)\}_{y \in N(x)}$ :

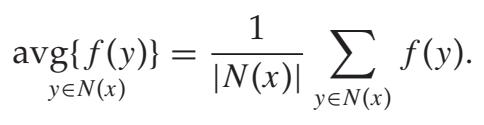

Other properties also follow. Assuming $f(x) \neq \bar{f}$ then

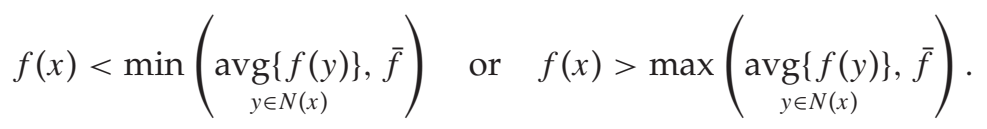

This implies that all maxima are greater than $\bar{f}$ and all minima are smaller than $\bar{f}$ (Codenotti and Margara, 1992).

An arbitrary landscape $(X, N, f)$ is not always elementary. However, even in this case, it is possible to characterize the function $f$ as the sum of elementary landscapes. In particular, if the neighborhood $N$ is symmetric, then it is possible to find an orthogonal basis composed of elementary functions. Thus, every discrete function (elementary or not) can be written as the sum of a set of elementary landscapes. The process of decomposing a landscape into its elementary components is what we call elementary landscape decomposition.

Such a decomposition could be useful from the theoretical and practical points of view. In theory, the landscape decomposition of a problem can be used to compute the exact expression for the autocorrelation functions, the autocorrelation coefficient, and the autocorrelation length (Angel and Zissimopoulos, 2000a). This information can potentially be used to estimate the performance of a local search method. Some researchers have studied the relationship between the performance of a local search and the autocorrelation coefficient (Angel and Zissimopoulos, 2000b). There exists a relationship between the autocorrelation length and the expected number of local optima of a problem (García-Pelayo and Stadler, 1997). In practice, the landscape decomposition together with Grover's wave equation can be used to compute the average value of the objective function in the neighborhood of a solution, which can be used as a base for new operators or algorithms (Sutton et al., 2010; Lu et al., 2010; Whitley and Sutton, 2009).

Finding the elementary landscape decomposition of a problem is not a trivial task. In general, it requires finding an orthogonal basis of eigenvectors of the neighborhood operator. In some cases, this is straightforward. For example, for the binary string representation and the bit-flip neighborhood, one such basis is the set of Walsh functions 
(Sutton et al., 2009). Using the Walsh decomposition one can show that MAX $k$-SAT is a superposition of $k$ elementary landscapes, and every NK-landscape is a superposition of $K+1$ elementary landscapes. But for other representations, finding the orthogonal basis of eigenvectors can be difficult (Angel and Zissimopoulos, 2000b).

This paper makes three fundamental contributions to research on elementary landscapes. First, new theoretical results are presented that generalize our understanding of elementary landscapes and their properties. Second, we develop a methodology based on linear algebra that can potentially be used to find a decomposition of a function into a linear combination of subfunctions, where each subfunction is elementary. Finally, we then use this methodology to prove that the subset sum problem is a superposition of two elementary landscapes, and the quadratic assignment problem is a superposition of three elementary landscapes. Showing that a problem is a superposition of elementary landscapes makes it possible to extend many calculations which can be done on elementary landscapes (such as computing neighborhood averages and the exact autocorrelation of the neighborhood structure) to these other landscapes which are not directly elementary.

The organization of the paper is as follows. In Section 2 we first review elementary landscapes, then present new theorems that provide the foundations needed to support the methods used later in this paper. Section 3 presents the proposed methodology and illustrates the explanations with a simple example, the subset sum problem. Section 4 illustrates the methodology in a complex example, the quadratic assignment problem (QAP). In Section 5 we present some limitations of the proposed methodology and, finally, Section 6 concludes the paper and proposes some lines of future research.

\section{Background and Theoretical Foundations}

In this section we present some fundamental results on landscapes theory. Most of the results presented here can be found in previous work (Reidys and Stadler, 2002). However, we highlight some observations that can be easily derived from well-known facts but are not present in the previous literature as far as we know.

Let $X$ be a finite set of solutions, $f: X \rightarrow \mathbb{R}$ be a real-valued function defined on $X$, and $N: X \rightarrow \mathcal{P}(X)$ the neighborhood operator. The pair $(X, N)$ is called configuration space and can be represented using a graph $G(X, E)$ in which $X$ is the set of vertices and a directed edge $(x, y)$ exists in $E$ if $y \in N(x)$ (Biyikoglu et al., 2007). We can represent the neighborhood operator by its adjacency matrix

$$
A_{x y}=\left\{\begin{array}{ll}
1 & \text { if } y \in N(x) \\
0 & \text { otherwise }
\end{array} .\right.
$$

The degree matrix $D$ is defined as the diagonal matrix

$$
D_{x y}=\left\{\begin{array}{ll}
|N(x)| & \text { if } x=y \\
0 & \text { otherwise }
\end{array}\right. \text {. }
$$

Any discrete function, $f$, defined over the set of candidate solutions can be characterized as a vector in $\mathbb{R}^{|X|}$. Using the graph representation of the configuration space, any function $f$ can be interpreted as a labeling of the nodes in the graph, where the label of node $x$ is $f(x)$. Any $|X| \times|X|$ matrix can be interpreted as a linear map that acts 
on vectors in $\mathbb{R}^{|X|}$. The Laplacian matrix of a neighborhood operator is defined as

$$
\Delta=A-D
$$

The Laplacian matrix acts on function $f$ as follows

$$
\Delta f=\left(\begin{array}{l}
\sum_{y \in N\left(x_{1}\right)}\left(f(y)-f\left(x_{1}\right)\right) \\
\sum_{y \in N\left(x_{2}\right)}\left(f(y)-f\left(x_{2}\right)\right) \\
\vdots \\
\sum_{y \in N\left(x_{|X|}\right)}\left(f(y)-f\left(x_{|X|}\right)\right)
\end{array}\right) .
$$

The component $x$ of this matrix-vector product can thus be written as:

$$
(\Delta f)(x)=\sum_{y \in N(x)}(f(y)-f(x))
$$

In this paper, we will restrict our attention to regular neighborhoods, where $|N(x)|=$ $d>0$ for a constant $d$, for all $x \in X$. When a neighborhood is regular, $\Delta=A-d I$. Stadler (1995) defines the class of elementary landscapes where the function $f$ is an eigenvector (or eigenfunction) of the Laplacian up to an additive constant. Formally, we have the following definition.

Definition 1 (ELEMENTARY Function And LANDSCAPE): Let $(X, N, f)$ be a landscape and $\Delta$ the Laplacian matrix of the neighborhood operator $N$. The function $f$ is said to be elementary if there exists a constant $b$, which we call offset, and an eigenvalue $\lambda$ of $\Delta$ such that $\Delta(f-b)=\lambda(f-b)$. The landscape itself is elementary if $f$ is elementary.

According to the previous definition, every elementary function, $f$, can be written as the sum of an eigenfunction of $\Delta, g$, and a constant $b$, that is, $f=g+b$. Taking into account basic results of linear algebra, it is not difficult to prove that if $f$ is elementary with eigenvalue $\lambda, a f+b$ is also elementary with the same eigenvalue $\lambda$. The next properties are a consequence of the particular characteristics of $\Delta$.

Proposition 1: Given the function $f: X \rightarrow \mathbb{R}$ and the Laplacian $\Delta$ defined on the regular neighborhood operator $N$, the following properties hold:

1. If $f$ is a constant function, that is, $f(x)=b \forall x \in X$ for a constant $b$, then $\Delta f=0$ and $f$ is an eigenfunction of $\Delta$ with eigenvalue $\lambda=0$.

2. If $f$ is elementary for the neighborhood $N$ with eigenvalue $\lambda$, then there exists a constant $b$ such that

$$
\underset{y \in N(x)}{\operatorname{avg}\{f(y)\}}=f(x)-\frac{\lambda}{d}(b-f(x)),
$$

where $d$ is the size of the neighborhood. 
PROOF: For the first property we can use Equation (7) and write:

$$
(\Delta f)(x)=\sum_{y \in N(x)}(f(y)-f(x))=\sum_{y \in N(x)}(b-b)=0 .
$$

This happens for each $x \in X$, so $\Delta f=0$ and it is an eigenfunction of $\Delta$ with eigenvalue 0 .

For the second property we again use Equation (7) to write:

$$
(\Delta f)(x)=\sum_{y \in N(x)}(f(y)-f(x))=\sum_{y \in N(x)} f(y)-d f(x) .
$$

Dividing by $d$ the previous equation we get:

$$
\frac{1}{d}(\Delta f)(x)=\underset{y \in N(x)}{\operatorname{avg}\{f(y)\}-f(x)}
$$

Since $f$ is elementary with eigenvalue $\lambda$, there exists a constant $b$ such that $\Delta(f-$ $b)=\lambda(f-b)$. Then, we can write the following with the help of Equation (9):

$$
\frac{1}{d}(\Delta(f-b))(x)=\frac{1}{d}(\Delta f)(x)=\underset{y \in N(x)}{\operatorname{avg}}\{f(y)\}-f(x)=\frac{\lambda}{d}(f(x)-b),
$$

where we used the first property to remove $b$ from the first member. We can rewrite the last two members as

$$
\underset{y \in N(x)}{\operatorname{avg}\{f(y)\}}=f(x)-\frac{\lambda}{d}(b-f(x)) .
$$

What is generally known as Grover's wave equation is just a particular instance of this more general result, for which Grover's equation $b=\bar{f}$, where is $\bar{f}$ the average of the function $f$ over the entire solution set $X$, that is, $\bar{f}=\left(\sum_{x \in X} f(x)\right) /|X|$. As far as we know, Equation (8) has not previously been reported in the literature. Its relevance comes from the fact that it is valid in all the regular neighborhoods (not only in the symmetric ones). Grover's wave equation can be stated as a special case of Proposition 1 in which the neighborhood is symmetric.

TheOrem 1 (Grover's WAVE EQUATION): The landscape $(X, N, f)$ with $N$ symmetric and regular is elementary if and only if the following expression holds:

$$
\underset{y \in N(x)}{\operatorname{avg}\{f(y)\}}=f(x)+\frac{k}{d}(\bar{f}-f(x)) \quad \forall x \in X,
$$

where $k$ is the additive inverse of the eigenvalue of $f$, that is, $k=-\lambda$. 
Grover's equation requires that the neighborhood be symmetric and regular. We say that a neighborhood $N$ is symmetric if for all $x, y \in X$ it holds that $y \in N(x)$ implies $x \in N(y)$, that is, if $y$ is a neighbor of $x$ then $x$ is a neighbor of $y$.

In Proposition 1 we proved that constant functions are eigenvectors of $\Delta$ with $\lambda=0$. Now we can ask the opposite: are all the eigenvectors of $\Delta$ with $\lambda=0$ constant functions? The general answer is no. However, as it is stated by Stadler (1996), if the neighborhood $N$ is connected then the multiplicity of the eigenvalue $\lambda=0$ is one, and this means that only constant functions are eigenvectors of $\Delta$. Thus, for connected neighborhoods the answer to the previous question is yes. We say that a neighborhood $N$ is connected if for each pair of solutions $x, y \in X$ we can find a finite sequence of solutions $x=x_{1}, x_{2}, \ldots, x_{q}=y$ such that $x_{i+1} \in N\left(x_{i}\right)$ for $i=1,2, \ldots, q-1$.

From Grover's wave equation we conclude that in an elementary landscape there exists a linear relationship between the average of the function in the neighborhood of a solution and the value of the function in that solution. We now ask if the linear relationship is a general characteristic of elementary landscapes. The following proposition positively answers this question.

Proposition 2: Let $(X, N, f)$ be a landscape where the neighborhood, $N$, is regular and symmetric. Then, $f$ is elementary if and only if there exist two constants $\alpha$ and $\beta$ such that:

$$
\underset{y \in N(x)}{\operatorname{avg}\{f(y)\}=\alpha f(x)+\beta \quad \forall x \in X .}
$$

PROOF: If the landscape is elementary, then Equation (11) follows from Theorem 1. Let us prove the reciprocal implication. We assume that Equation (11) holds. Then, we can multiply both members by $d$ to write:

$$
\sum_{y \in N(x)} f(y)=d \alpha f(x)+d \beta=d f(x)+d(\alpha-1) f(x)+d \beta
$$

If we subtract $d f(x)$ we have:

$$
\sum_{y \in N(x)} f(y)-d f(x)=d(\alpha-1) f(x)+d \beta .
$$

At this point we must consider two cases. First, let us consider the case in which $\alpha=1$, then we can write the previous equation in vector form as:

$$
\Delta f=d \beta\left(\begin{array}{c}
1 \\
1 \\
\vdots \\
1
\end{array}\right) .
$$


Multiplying by the row vector $(1,1, \ldots, 1)$ in both members we get:

$$
(1,1, \ldots, 1) \Delta f=d \beta(1,1, \ldots, 1)\left(\begin{array}{c}
1 \\
1 \\
\vdots \\
1
\end{array}\right)=d \beta|X|
$$

However, due to the symmetry of the neighborhood, it is possible to write:

$$
d \beta|X|=((1,1, \ldots, 1) \Delta f)^{T}=f^{T} \Delta\left(\begin{array}{c}
1 \\
1 \\
\vdots \\
1
\end{array}\right)=0
$$

which implies $\beta=0$ since $d$ and $|X|$ are greater than zero. Then $\Delta f=0$ and $f$ is an elementary landscape with $\lambda=0$. This does not necessarily mean that $f$ is a constant, since the neighborhood is not necessarily connected. If the neighborhood is connected, $f$ must be a constant function.

Now, let us consider the case in which $\alpha \neq 1$. Then, we can write in vector form:

$$
\Delta f=d(\alpha-1) f+d \beta\left(\begin{array}{c}
1 \\
1 \\
\vdots \\
1
\end{array}\right) \text {. }
$$

Taking into account the results of Proposition 1 and the definition of an elementary landscape we can write:

$$
\Delta\left(f+\frac{\beta}{\alpha-1}\left(\begin{array}{c}
1 \\
1 \\
\vdots \\
1
\end{array}\right)\right)=\Delta f=d(\alpha-1)\left(f+\frac{\beta}{\alpha-1}\left(\begin{array}{c}
1 \\
1 \\
\vdots \\
1
\end{array}\right)\right)
$$

and $f$ is elementary with eigenvalue $\lambda=d(\alpha-1)$.

The previous result provides a useful characterization of elementary landscapes that allows us to simplify the proof that a given landscape is elementary (or not). Although the result can be easily derived, to the best of our knowledge it has not been reported in the previous literature and it has not been used to check if a landscape is elementary. 
When $f$ is not an elementary landscape, Equation (11) does not hold, but we can find a generalization of the equation that does hold if $f$ is the sum of $n$ elementary landscapes. This general expression is presented in the following.

THEOREM 2: Let $(X, N, f)$ be a landscape in which the neighborhood, $N$, is regular and symmetric. Then, $f$ is the sum of $n$ nonconstant elementary landscapes $f_{i}$ if and only if there exist some constants $\alpha_{i}$ for $i=0,1, \ldots, n$ such that

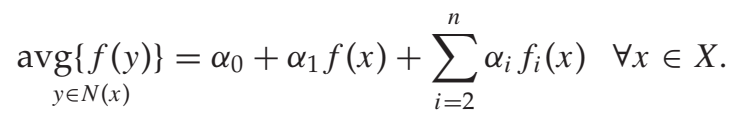

PROOF: We can prove this by induction on $n$. In the base case, $n=1$, Proposition 2 holds and the statement is true. For the inductive step, let us assume that the statement is true for $n-1$ and let us prove the result for $n$.

Assume the function $f$ is the sum of $n$ elementary landscapes $f_{i}$, that is:

$$
f=\sum_{i=1}^{n} f_{i}
$$

If we subtract $f_{n}$ in the previous equality, then $f-f_{n}$ is the sum of $n-1$ elementary landscapes. We can apply the inductive hypothesis to compute the average value in the neighborhood of an arbitrary solution $x$. That is, a set of constants $\alpha_{i}$ exists such that:

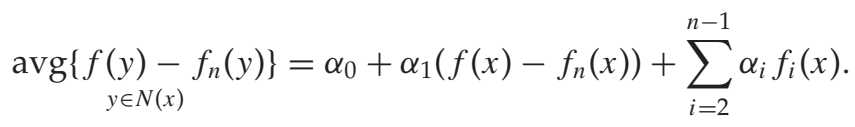

Since $f_{n}$ is an elementary landscape, according to Proposition 2 we can write $\operatorname{avg}\left\{f_{n}(y)\right\}_{y \in N(x)}=\beta_{0}+\beta_{1} f_{n}(x)$, and the previous expression can be written as:

$$
\begin{aligned}
\underset{y \in N(x)}{\operatorname{avg}\{f(y)\}} & =\alpha_{0}+\alpha_{1}\left(f(x)-f_{n}(x)\right)+\sum_{i=2}^{n-1} \alpha_{i} f_{i}(x)+\underset{y \in N(x)}{\operatorname{avg}\left\{f_{n}(y)\right\}} \\
& =\alpha_{0}+\alpha_{1}\left(f(x)-f_{n}(x)\right)+\sum_{i=2}^{n-1} \alpha_{i} f_{i}(x)+\beta_{0}+\beta_{1} f_{n}(x) \\
& =\left(\alpha_{0}+\beta_{0}\right)+\alpha_{1} f(x)+\sum_{i=2}^{n-1} \alpha_{i} f_{i}(x)+\left(\beta_{1}-\alpha_{1}\right) f_{n}(x)
\end{aligned}
$$

and Equation (12) holds for $n$.

Let us now prove the reciprocal implication. Let us assume that Equation (12) holds for a given $f$, where all $f_{i}$ are elementary functions. Since $f_{n}$ is a nonconstant elementary function, we can apply Proposition 2 and write $\operatorname{avg}\left\{f_{n}(y)\right\}_{y \in N(x)}=\beta_{0}+\beta_{1} f_{n}(x)$ with 
$\beta_{1} \neq 0$. Then Equation (12) can be rewritten as:

$$
\begin{aligned}
\underset{y \in N(x)}{\operatorname{avg}\{f(y)\}} & =\alpha_{0}+\alpha_{1} f(x)+\sum_{i=2}^{n-1} \alpha_{i} f_{i}(x)+\frac{\alpha_{n}}{\beta_{1}}\left(\underset{y \in N(x)}{\left.\operatorname{avg}\left\{f_{n}(y)\right\}-\beta_{0}\right)}\right. \\
& =\alpha_{0}+\alpha_{1} f(x)+\sum_{i=2}^{n-1} \alpha_{i} f_{i}(x)+\operatorname{avg}\left\{\alpha _ { n } \left(\underset{n}{\left.\left(f_{n}(y)-\beta_{0}\right) / \beta_{1}\right\}}\right.\right.
\end{aligned}
$$

In order to simplify the expressions, let us define the function $g=\alpha_{n}\left(f_{n}-\beta_{0}\right) / \beta_{1}$. We can rewrite the previous expression in the following way:

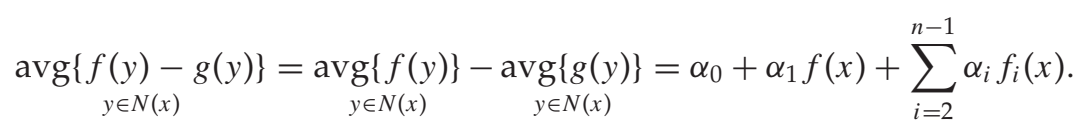

Using the inductive hypothesis, $f-g$ is the sum of $n-1$ elementary landscapes and this implies that $f$ is the sum of $n$ elementary landscapes since $g$ is also an elementary landscape with the same eigenvalue as $f_{n}$.

The previous result allows us to compute the average value of the objective function in the neighborhood of a given solution $x$ from the value of the objective function $f$ and its elementary components $f_{i}$ in $x$. This average value could be useful in practice for guiding a search method, but it requires knowing the elementary components of the objective function. Thus, we can state that the decomposition of a given function into elementary components could be important in practice. We previously highlighted that this decomposition is also useful in theory (for computing the autocorrelation coefficient). Now, let us prove that any objective function can be written as the sum of elementary landscapes when the neighborhood is symmetric.

TheOREM 3 (ElEMENTARy LANDSCAPE DeCOMPOSition): Let $(X, N, f)$ be a landscape where the neighborhood, $N$, is symmetric. Then, there exist n elementary landscapes with $1 \leq n \leq|X|$ such that $f$ can be written as the sum of all of them.

PROOF: From linear algebra we know that if a square real matrix $\Delta$ of size $|X|$ is symmetric, then there exists an orthogonal basis of the vector space $\mathbb{R}^{|X|}$ that is composed of eigenvectors of $\Delta$. Then, we can write every vector of $\mathbb{R}^{|X|}$ as the weighted sum of the vectors in the orthogonal basis. This means that for any symmetric neighborhood $N$ it is possible to find an orthogonal basis composed of elementary functions. Then, any function $f$ can be written as the weighted sum of a set of elementary landscapes.

The next section presents a methodology for finding the decomposition of an objective function into a superposition of elementary landscapes.

\section{Algebra-based Methodology}

Given a function $f$ and a neighborhood $N$ (a landscape), the problem is to find a decomposition of the function into multiple elementary landscapes. As we previously stated, for a symmetric neighborhood $N$, there exists an orthogonal basis composed of 
elementary landscapes. Let us denote this basis with $\theta_{\lambda, i}$ where $\lambda$ is the eigenvalue of the vector (function) and $i$ is an index to distinguish the different vectors with the same eigenvalue. Then a Fourier expansion of $f$ is

$$
f=\sum_{\lambda} \sum_{i} a_{\lambda, i} \theta_{\lambda, i}
$$

where the values $a_{\lambda, i}=\left\langle\theta_{\lambda, i}, f\right\rangle$ are the Fourier coefficients. Using this Fourier expansion, it is possible to compute the landscape decomposition by summing the terms with the same eigenvalue. Each elementary component can be computed as

$$
f_{\lambda}=\sum_{i} a_{\lambda, i} \theta_{\lambda, i}
$$

A special case is that of $f_{0}$, the elementary landscape with $\lambda=0$. We assume that the neighborhood is connected. Then, $f_{0}$ is the constant value $\bar{f}$, and it could be added to any of the other elementary components and still the landscape would remain elementary.

Equation (14) can be used when an orthogonal basis of eigenvectors is known for the neighborhood. This happens, for example, in the case of binary strings with the bit-flip neighborhood. An appropriate basis for this neighborhood is the set of Walsh functions (Sutton et al., 2009). But in general such a basis is not known or, when it is known, it is not easy to compute the Fourier coefficients. The methodology we present here is useful under these situations.

The methodology consists of analyzing instances of the problem that are small enough that it is possible to enumerate the Laplacian matrix $\Delta$. This way, it is possible to obtain a basis of $\mathbb{R}^{|X|}$ composed of eigenvectors of $\Delta$. With the help of this basis we can decompose the objective function into subfunctions which are elementary. Then, a detailed study of the elementary components can reveal the general definition of these components in any general (and larger) instance of the problem.

We have identified five steps for applying the methodology:

1. Rewrite the objective function as a linear combination of the so-called basic functions, denoted with $\varphi$.

2. Compute $\Delta$ and $\varphi$ for small instances.

3. Compute the projections of $\varphi$ in the eigenspaces of $\Delta$.

4. Analyze the projections and propose elementary components.

5. Check the landscape decomposition in the general case.

In the following we explain in detail the operations involved in each step and we illustrate the application of this methodology with a landscape decomposition for the subset sum problem (Garey and Johnson, 1979). Given a set of integers $S=\left\{s_{1}, s_{2}, \ldots, s_{n}\right\}$, the problem consists of finding a nonempty subset of $S$ whose sum is $C$ (if any). This problem can be transformed as a minimization problem with objective function

$$
f(x)=\left(\sum_{i=1}^{n} s_{i} x_{i}-C\right)^{2}
$$


where $x_{i} \in\{0,1\}$ are the decision variables of the problem. Thus, the size of the solution space $X$ is $2^{n}$, and the neighborhood is the bit-flip neighborhood: two solutions are neighbors if one of them can be obtained by changing the value of one decision variable $x_{i}$ in the other one.

\subsection{Step 1: Rewrite the Objective Function}

In order to analyze the elementary components of the objective function, it is useful to separate the definition of the objective function into (1) the information that is particular to a given instance (the data of the instance); and (2) the general relationships that characterize the class of the problem. We are interested in linear combinations of functions, called basic functions, where the coefficients of the functions are the data of the particular instances. Note that any linear combination of elementary functions with the same characteristic constant $k$ is also an elementary function. Then we reduce the analysis of the general objective function containing instance information to the analysis of a family of basic functions that do not depend on the instance data. We denote these basic functions with the letter $\varphi$ and we use subscripts and superscripts to parameterize the basic functions.

We illustrate this first step using the subset sum problem. We can rewrite Equation (15) in the following way:

$$
\begin{aligned}
f(x) & =\left(\sum_{i=1}^{n} s_{i} x_{i}-C\right)^{2}=\sum_{i, j=1}^{n} s_{i} s_{j} x_{i} x_{j}-2 C \sum_{i=1}^{n} s_{i} x_{i}+C^{2} \\
& =\sum_{i, j=1}^{n} s_{i} s_{j} \varphi_{i j}(x)-2 C \sum_{i=1}^{n} s_{i} \varphi_{i i}(x)+C^{2}
\end{aligned}
$$

where we write $f$ as a linear combination of the parameterized functions $\varphi_{i j}(x)=x_{i} x_{j}$. All the information related to each particular instance is focused on the weights (the coefficients) of this linear combination. Thus, we only have to study the family of basic functions $\varphi_{i j}$. Using the landscape decomposition of these basic functions, it is possible to compute the landscape decomposition of $f$ for any instance of the problem (set $S$ ).

\subsection{Step 2: Compute $\Delta$ and $\varphi$ for Small Instances}

Recall that we are dealing with a problem class. This means that we are analyzing a (possibly infinite) set of problem instances at the same time. These instances can have different sizes, and by size we mean the cardinality of the solution space $X$. For example, in the subset sum problem we have

$$
|X|=2^{n}
$$

where $n$ is the number of integers in the set $S$.

In the second step of this methodology we need to explicitly compute the Laplacian matrix $\Delta$ and we explicitly represent the basic functions $\varphi$ using a vector. Thus, the larger the cardinality of $X$, the larger the size of $\Delta$ and $\varphi$. Since we have to numerically operate with $\Delta$ and $\varphi$, it is preferable to work with small solution spaces. The number of solution spaces required depends on the number of elementary components of $\varphi$. A good rule of thumb here is to use the smaller solution spaces for which the Laplacian matrix has a size that affords its use with a computer algebra system. 
In the subset sum problem, we can use for the cardinality of the subset $S$ the values $n=2,3,4$ which are related to solution spaces with sizes $|X|=2^{2}, 2^{3}, 2^{4}$. If we sort the solutions in lexicographical order, the Laplacian matrices for these solution spaces are the following:

$$
\Delta_{2}=\left(\begin{array}{cccc}
-2 & 1 & 1 & 0 \\
1 & -2 & 0 & 1 \\
1 & 0 & -2 & 1 \\
0 & 1 & 1 & -2
\end{array}\right)
$$

$$
\Delta_{4}=\left(\begin{array}{cccccccccccccccc}
-4 & 1 & 1 & 0 & 1 & 0 & 0 & 0 & 1 & 0 & 0 & 0 & 0 & 0 & 0 & 0 \\
1 & -4 & 0 & 1 & 0 & 1 & 0 & 0 & 0 & 1 & 0 & 0 & 0 & 0 & 0 & 0 \\
1 & 0 & -4 & 1 & 0 & 0 & 1 & 0 & 0 & 0 & 1 & 0 & 0 & 0 & 0 & 0 \\
0 & 1 & 1 & -4 & 0 & 0 & 0 & 1 & 0 & 0 & 0 & 1 & 0 & 0 & 0 & 0 \\
1 & 0 & 0 & 0 & -4 & 1 & 1 & 0 & 0 & 0 & 0 & 0 & 1 & 0 & 0 & 0 \\
0 & 1 & 0 & 0 & 1 & -4 & 0 & 1 & 0 & 0 & 0 & 0 & 0 & 1 & 0 & 0 \\
0 & 0 & 1 & 0 & 1 & 0 & -4 & 1 & 0 & 0 & 0 & 0 & 0 & 0 & 1 & 0 \\
0 & 0 & 0 & 1 & 0 & 1 & 1 & -4 & 0 & 0 & 0 & 0 & 0 & 0 & 0 & 1 \\
1 & 0 & 0 & 0 & 0 & 0 & 0 & 0 & -4 & 1 & 1 & 0 & 1 & 0 & 0 & 0 \\
0 & 1 & 0 & 0 & 0 & 0 & 0 & 0 & 1 & -4 & 0 & 1 & 0 & 1 & 0 & 0 \\
0 & 0 & 1 & 0 & 0 & 0 & 0 & 0 & 1 & 0 & -4 & 1 & 0 & 0 & 1 & 0 \\
0 & 0 & 0 & 1 & 0 & 0 & 0 & 0 & 0 & 1 & 1 & -4 & 0 & 0 & 0 & 1 \\
0 & 0 & 0 & 0 & 1 & 0 & 0 & 0 & 1 & 0 & 0 & 0 & -4 & 1 & 1 & 0 \\
0 & 0 & 0 & 0 & 0 & 1 & 0 & 0 & 0 & 1 & 0 & 0 & 1 & -4 & 0 & 1 \\
0 & 0 & 0 & 0 & 0 & 0 & 1 & 0 & 0 & 0 & 1 & 0 & 1 & 0 & -4 & 1 \\
0 & 0 & 0 & 0 & 0 & 0 & 0 & 1 & 0 & 0 & 0 & 1 & 0 & 1 & 1 & -4
\end{array}\right) .
$$


Now we need a vector representation of the basic functions. Usually not all the basic functions are needed, since some of them are equivalent. We say that two basic functions $\varphi$ and $\varphi^{\prime}$ are equivalent if there exists an automorphism $\pi: X \rightarrow X$ of the graph $G$ induced by the configuration space such that $\varphi \circ \pi=\varphi^{\prime}$. In other words, we say that two basic functions are equivalent if they are essentially the same function seen from a different point of view of the graph. We can partition the family of basic functions according to the previous equivalence relation and study only one basic function from each equivalence class.

In the subset sum problem, the basic functions $\varphi_{i j}$ can be partitioned into two equivalence classes: those in which $i \neq j$ and those for which $i=j$. In effect, for a pair of basic functions $\varphi_{i j}$ and $\varphi_{i^{\prime} j^{\prime}}$ in which $i \neq j$ and $i^{\prime} \neq j^{\prime}$ an automorphism for which $\varphi_{i^{\prime} j^{\prime}} \circ \pi=\varphi_{i j}$ is:

$$
\begin{aligned}
\pi: X \rightarrow X \\
\pi(x) \mapsto y
\end{aligned}
$$

where $y_{i}=x_{i^{\prime}}, y_{i^{\prime}}=x_{i}, y_{j}=x_{j^{\prime}}, y_{j^{\prime}}=x_{j}$ and $y_{k}=x_{k}$ for $k \neq i, i^{\prime}, j, j^{\prime}$. For a pair of basic functions $\varphi_{i i}$ and $\varphi_{i^{\prime} i^{\prime}}$ an automorphism for which $\varphi_{i^{\prime} i^{\prime}} \circ \pi=\varphi_{i i}$ is $\pi$ where $\pi(x)=y$ and $y_{i}=x_{i \prime}, y_{i^{\prime}}=x_{i}$, and $y_{k}=x_{k}$ for $k \neq i, i^{\prime}$. On the other hand, the basic functions $\varphi_{i i}$ and $\varphi_{i j}$ cannot be equivalent if $j \neq i$ since both functions have a different number of solutions with value 1 . Having the same number of solutions with a given function value is a necessary condition for equivalence.

As a sample of the two equivalence classes in which the basic functions can be partitioned, let us study the functions $\varphi_{12}$ and $\varphi_{11}$ for the cardinalities of $S$ used before $n=2,3,4$. In vector form these basic functions are:

$$
\begin{aligned}
& \vec{\varphi}_{11}=\left(\begin{array}{llll}
0 & 1 & 0 & 1
\end{array}\right)^{T} \\
& \vec{\varphi}_{12}=\left(\begin{array}{llll}
0 & 0 & 0 & 1
\end{array}\right)^{T} \text { for } n=2 \\
& \vec{\varphi}_{11}=\left(\begin{array}{llllllll}
0 & 1 & 0 & 1 & 0 & 1 & 0 & 1
\end{array}\right)^{T} \\
& \vec{\varphi}_{12}=\left(\begin{array}{llllllll}
0 & 0 & 0 & 1 & 0 & 0 & 0 & 1
\end{array}\right)^{T} \text { for } n=3 \\
& \vec{\varphi}_{11}=\left(\begin{array}{llllllllllllllll}
0 & 1 & 0 & 1 & 0 & 1 & 0 & 1 & 0 & 1 & 0 & 1 & 0 & 1 & 0 & 1
\end{array}\right)^{T} \\
& \vec{\varphi}_{12}=\left(\begin{array}{llllllllllllllll}
0 & 0 & 0 & 1 & 0 & 0 & 0 & 1 & 0 & 0 & 0 & 1 & 0 & 0 & 0 & 1
\end{array}\right)^{T} \text { for } n=4 \text {. }
\end{aligned}
$$

We use the two notations $\varphi$ and $\vec{\varphi}$ (with the corresponding subscripts and superscripts) to represent the basic functions. However, we use vector notation when we want to highlight the vector nature of the function.

\subsection{Step 3: Compute the Projections of $\varphi$ in the Eigenspaces of $\Delta$}

In this step we decompose the basic functions into their elementary components for the instance sizes considered in the previous step. In order to do this, we first compute the eigenvalues of the Laplacian and an orthonormal vector basis composed of eigenvectors, 
also known as an eigensystem. Let $\vec{e}_{\lambda, i}$ denote a basis vector having eigenvalue $\lambda$. The subscript $i$ is used to distinguish between the vectors with the same eigenvalue. Once we know the basis, we compute the coordinates of the basic functions in this new basis, that is: $a_{\lambda, i}=\left\langle\vec{e}_{\lambda, i}, \vec{\varphi}\right\rangle$. The vector $a_{\lambda, i} \vec{e}_{\lambda, i}$ is the projection of $\vec{\varphi}$ onto the vector $\vec{e}_{\lambda, i}$. Finally, we compute the projections into the different eigenspaces of $\Delta$ by summing all the projections of $\vec{\varphi}$ onto vectors with the same eigenvalue. We denote these projections with $\vec{\phi}^{\lambda}$. As a result, we obtain several vectors (or functions), each one corresponding to an elementary function.

We should note here that although dealing with orthonormal bases of eigenvectors of the Laplacian is difficult in general (this was one of the arguments to develop this methodology) finding a basis in this case is not difficult, since we are working with small instances of the problem. In fact, this step can be done automatically without human intervention.

Now let us illustrate this step with the subset sum problem. First, we obtain the eigensystem using a computer algebra system. We show here only an eigensystem for $\Delta_{2} . \Delta_{2}$ has three eigenvalues: $-4,-2$, and 0 .

$$
\vec{e}_{-4,1}=\frac{1}{2}\left(\begin{array}{c}
1 \\
-1 \\
-1 \\
1
\end{array}\right) \quad \vec{e}_{-2,1}=\frac{1}{\sqrt{2}}\left(\begin{array}{c}
-1 \\
0 \\
0 \\
1
\end{array}\right) \quad \vec{e}_{-2,2}=\frac{1}{\sqrt{2}}\left(\begin{array}{c}
0 \\
-1 \\
1 \\
0
\end{array}\right) \quad \vec{e}_{0,1}=\frac{1}{2}\left(\begin{array}{l}
1 \\
1 \\
1 \\
1
\end{array}\right)
$$

Now, we can compute the Fourier coefficients $a_{\lambda, i}$, and the projections $\vec{\phi}^{\lambda}$. For $\varphi_{11}$ and $n=2$ we obtain: $a_{-4,1}=0, a_{-2,1}=1 / \sqrt{2}, a_{-2,2}=-1 / \sqrt{2}$ and $a_{0,1}=1$. For $\varphi_{12}$ and $n=2$ we obtain: $a_{-4,1}=1 / 2, a_{-2,1}=1 / \sqrt{2}, a_{-2,2}=0$ and $a_{0,1}=1 / 2$. Then the projections of these functions into the eigenspaces of $\Delta_{2}$ are:

$$
\begin{gathered}
\vec{\phi}_{11}^{-4}=0 ; \quad \vec{\phi}_{11}^{-2}=\frac{1}{2}\left(\begin{array}{c}
-1 \\
1 \\
-1 \\
1
\end{array}\right) ; \quad \vec{\phi}_{11}^{0}=\frac{1}{2}\left(\begin{array}{l}
1 \\
1 \\
1 \\
1
\end{array}\right) \\
\vec{\phi}_{12}^{-4}=\frac{1}{4}\left(\begin{array}{c}
1 \\
-1 \\
-1 \\
1
\end{array}\right) ; \quad \vec{\phi}_{12}^{-2}=\frac{1}{2}\left(\begin{array}{c}
-1 \\
0 \\
0 \\
1
\end{array}\right) ; \quad \vec{\phi}_{12}^{0}=\frac{1}{4}\left(\begin{array}{l}
1 \\
1 \\
1 \\
1
\end{array}\right) .
\end{gathered}
$$

We observe that Equations (24) and (25) are the elementary landscape decomposition of $\varphi_{11}$ and $\varphi_{12}$, respectively, that is, $\varphi_{11}=\phi_{11}^{-2}+\phi_{11}^{0}$ and $\varphi_{12}=\phi_{12}^{-4}+\phi_{12}^{-2}+\phi_{12}^{0}$. The 
previous decomposition shows that $\varphi_{11}$ is an elementary landscape and $\varphi_{12}$ is the sum of two elementary landscapes (we can ignore the constant component $\phi^{0}$ in all the cases).

If we make the same computations for $\Delta_{3}$, we obtain the following decomposition:

$$
\begin{aligned}
\vec{\phi}_{11}^{-2} & =\frac{1}{2}(-1,1,-1,1,-1,1,-1,1)^{T} \\
\vec{\phi}_{12}^{-4} & =\frac{1}{4}(1,-1,-1,1,1,-1,-1,1)^{T} \\
\vec{\phi}_{12}^{-2} & =\frac{1}{2}(-1,0,0,1,-1,0,0,1)^{T}
\end{aligned}
$$

where we only show the nonzero projections that are not constant. Finally, for $\Delta_{4}$ we obtain the following decomposition:

$$
\begin{aligned}
\vec{\phi}_{11}^{-2} & =\frac{1}{2}(-1,1,-1,1,-1,1,-1,1,-1,1,-1,1,-1,1,-1,1)^{T} \\
\vec{\phi}_{12}^{-4} & =\frac{1}{4}(1,-1,-1,1,1,-1,-1,1,1,-1,-1,1,1,-1,-1,1)^{T} \\
\vec{\phi}_{12}^{-2} & =\frac{1}{2}(-1,0,0,1,-1,0,0,1,-1,0,0,1,-1,0,0,1)^{T} .
\end{aligned}
$$

\subsection{Step 4: Analyze the Projections and Propose Elementary Components}

Once we know how the basic functions of small instances can be decomposed into elementary landscapes, we have to generalize the results to larger instances.

Unlike Steps 2 and 3, which can be mechanically accomplished, this step requires human intervention. A person must analyze the decomposition obtained in Step 3 and generalize the results to propose a general decomposition for each basic function $\varphi$. Nevertheless, there are some mathematical tools that can help in this task. In the following we detail these tools and illustrate their use.

1. Given an elementary landscape, it is possible to multiply and sum any real value $c \in \mathbb{R}$ with the components; the landscape remains elementary. In our decomposition of the subset sum problem, we can remove the constants that multiply the vector in the basic function decomposition. For $n=3$ this gives:

$$
\begin{aligned}
& \vec{\phi}_{11}^{-2}=(-1,1,-1,1,-1,1,-1,1)^{T} \\
& \vec{\phi}_{12}^{-4}=(1,-1,-1,1,1,-1,-1,1)^{T} \\
& \vec{\phi}_{12}^{-2}=(-1,0,0,1,-1,0,0,1)^{T} .
\end{aligned}
$$

2. The number of elementary landscapes in the small instances and their eigenvalues can be a clue to determine how many elementary landscapes make up the general decomposition and which are the eigenvalues for these elementary components. In general, the eigenvalues can be different for the different instances considered, since the eigenvalues could depend on the problem size. In our example, for $n=2,3,4$ there is only one elementary component with eigenvalue $\lambda=-2$ for 


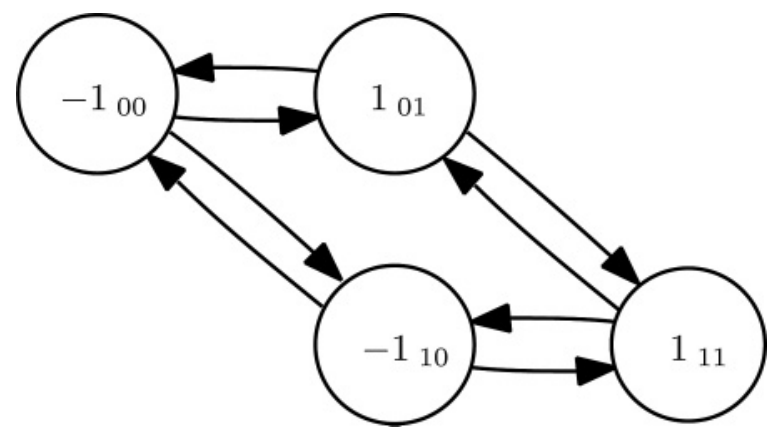

Figure 1: Graph $G=(X, E)$ for $n=2$. We label the nodes with $\phi(x)_{x}$. The equivalence class [00] contains 00 and 10 which both have evaluation -1 .

$\varphi_{11}$ and two elementary components for $\varphi_{12}$ with eigenvalues $\lambda=-2,-4$. Thus, we conjecture that in the general case, $\varphi_{11}$ is an elementary component and $\varphi_{12}$ can be decomposed as two elementary components with eigenvalues $\lambda=-2$ and $\lambda=-4$, respectively.

3. We can use the underlying graph of the landscape $G=(X, E)$ as a tool in the analysis of the landscape decomposition. To this aim we need to label the nodes of the graph with the values of the function $\phi$. Then, we construct a new graph by grouping together all the nodes that we consider equivalent according to the function value and graph structure. Equivalent now means not only to have the same function value, but their neighbors must also be equivalent. In formal terms, we say that two nodes $x$ and $y$ in the graph are equivalent for function $\phi$, and we denote it with $x \sim_{\phi} y$ when there exists an automorphism $\pi$ of the graph such that $\pi(x)=y$ and $\phi \circ \pi=\phi$. That is, in a graph labeled with function $\phi$, the nodes $x$ and $y$ cannot be distinguished. With this equivalence relationship the new graph is $G / \sim_{\phi}=\left(X / \sim_{\phi}, E / \sim_{\phi}\right)$ where $X / \sim_{\phi}$ is the quotient set of $X$ by $\sim_{\phi}$ and $([x],[y]) \in E / \sim_{\phi}$ if $(x, y) \in E$. The set $X / \sim_{\phi}$ is the set of equivalence classes in set $X$, and we use $[x]$ to denote the equivalence class containing $x$. In $G / \sim_{\phi}$ we label each node $[x]$ with the value $\phi(x)$. In addition, for this graph we also label each edge ([x], $[y])$ with the number of edges in the original graph $G$ of the form $(x, z)$ where $z \in[y]$. In other words, the label of $([x],[y])$ is the number of neighbors that any element in $[x]$ has with function value $\phi(y)$. This graph must be constructed for each elementary component $\phi$ of each basic function $\varphi$ in all the small instances considered. In the following we call this graph the reduced graph for function $\phi$.

Let us illustrate the graph construction with $\phi_{11}^{-2}$ for $n=2$. The original labeled graph is the one shown in Figure 1, where we also show the solution $x$ with the function value $\phi(x)$. It is not difficult to see in this case that nodes 00 and 10 are equivalent and the same is true for 01 and 11 . Then the reduced graph $G / \sim_{\phi_{11}^{-2}}$ is the one shown in Figure 2.

We have computed the reduced graph for all the $\phi$ functions and we show them in Figures 3, 4, and 5.

The reduced graphs can help in identifying features of the elementary components in order to generalize their definition. In order to define the general elementary 


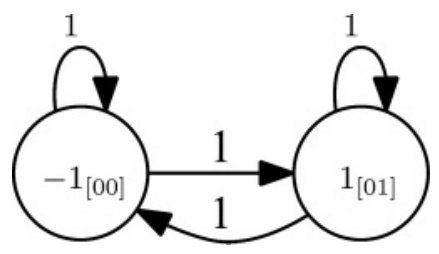

Figure 2: Graph $G / \sim_{\phi_{11}^{-2}}$ for $n=2$. We label the nodes with $\phi([x])_{[x]}$.
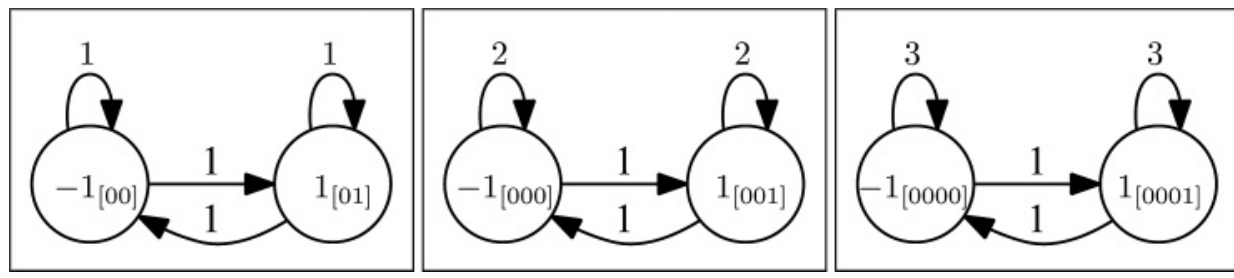

Figure 3: Graph $G / \sim_{\phi_{11}^{-2}}$ for $n=2,3$, 4. We label the nodes with $\phi([x])_{[x]}$.

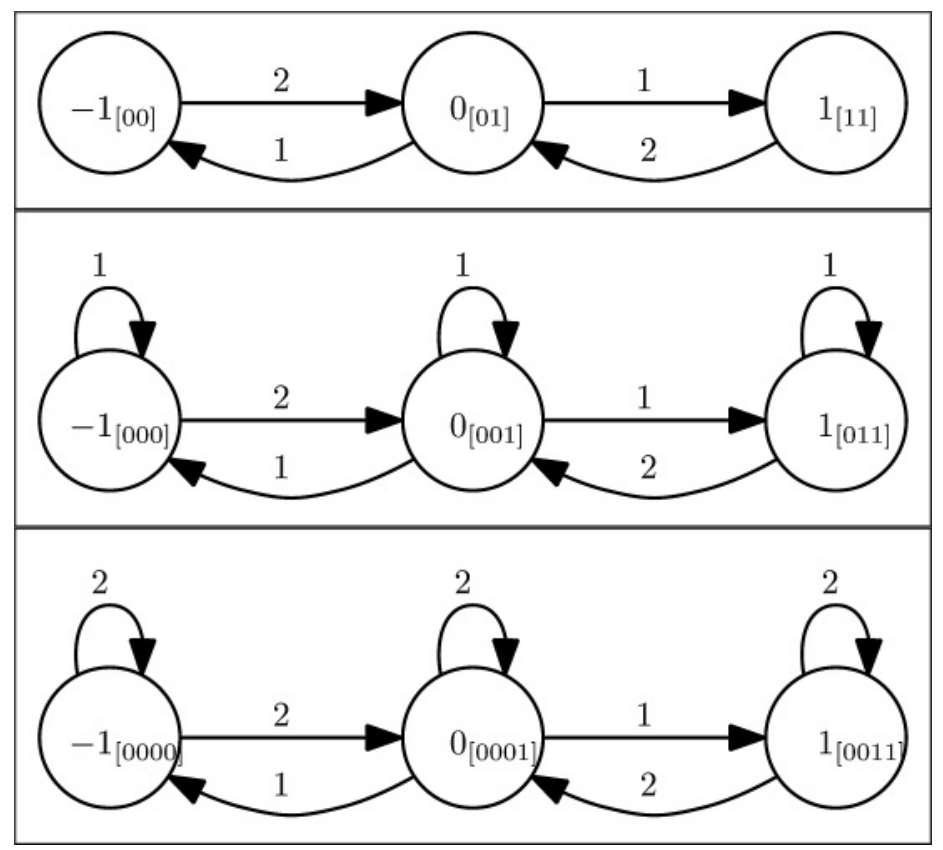

Figure 4: Graph $G / \sim_{\phi_{12}^{-2}}$ for $n=2,3$, 4. We label the nodes with $\phi([x])_{[x]}$.

components $\phi$ we first need to recognize this elementary component among the $\phi$ functions of the small instances considered with different sizes. This can be done by grouping together the $\phi$ functions of the different instances with some common feature. Then, we conjecture that these functions can be generalized to a most general elementary 


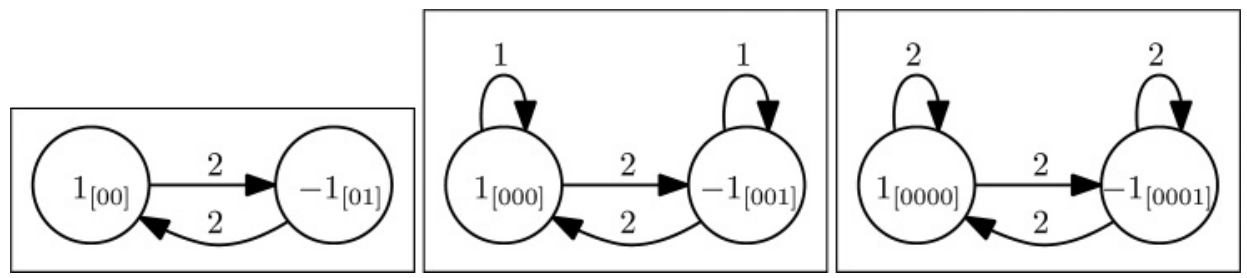

Figure 5: Graph $G / \sim_{\phi_{12}^{-4}}$ for $n=2,3$, 4. We label the nodes with $\phi([x])_{[x]}$.

Table 1: Equivalence classes for the subset sum problem with function $\phi_{12}^{-2}$.

\begin{tabular}{|c|c|c|c|c|c|c|c|c|}
\hline \multicolumn{3}{|c|}{$n=2$} & \multicolumn{3}{|c|}{$n=3$} & \multicolumn{3}{|c|}{$n=4$} \\
\hline [00] & [01] & [11] & [000] & [001] & [011] & [0000] & [0001] & [0011] \\
\hline \multirow[t]{8}{*}{00} & 01 & 11 & 000 & 001 & 011 & 0000 & 0001 & 0011 \\
\hline & 10 & & 100 & 010 & 111 & 0100 & 0010 & 0111 \\
\hline & & & & 101 & & 1000 & 0101 & 1011 \\
\hline & & & & 110 & & 1100 & 0110 & 1111 \\
\hline & & & & & & & 1001 & \\
\hline & & & & & & & 1010 & \\
\hline & & & & & & & 1101 & \\
\hline & & & & & & & 1110 & \\
\hline
\end{tabular}

component. After that, we propose the generalization by observing the classes of equivalence in $X / \sim_{\phi}$. Let us illustrate this with our example.

In Figures 3, 4, and 5 we have grouped together the $\phi$ functions according to their eigenvalues. As we previously argued, in this example it seems that $\varphi_{11}$ is elementary and $\varphi_{12}$ can be decomposed into two elementary components with eigenvalues $\lambda=-2$ and $\lambda=-4$. Then, it is reasonable to think that the functions $\phi_{11}^{-2}$ for the different values of $n$ are elementary, and the same holds for $\phi_{12}^{-2}$ and $\phi_{12}^{-4}$. In this case, the grouping seems evident. If the eigenvalues were different for the different sizes of the problem, then the grouping would not be so evident.

The next step is, then, to propose a generalization for the grouped functions. The simplest case is that of $\phi_{11}^{-2}$, since its elementariness implies the elementariness of $\varphi_{11}$, so we should be able to write $\phi_{i i}^{-2}$ as a function of $\varphi_{i i}$. A possible generalization of this function is $\phi_{i i}^{-2}=2 \varphi_{i i}-1=2 x_{i}-1$. In the fifth step we will check if this function is elementary or not.

Let us follow with $\phi_{12}^{-2}$. In Figure 4 we have not shown the equivalence classes. They are shown in Table 1 . In the table we show the values of $x_{i}$ in big-endian order $\left(x_{n}\right.$ first and $x_{1}$ last). A closer look to the equivalence classes suggests that $\phi_{12}^{-2}=-1$ if $x_{1}=x_{2}=0$, $\phi_{12}^{-2}=1$ if $x_{1}=x_{2}=1$, and $\phi_{12}^{-2}=0$ if $x_{1} \neq x_{2}$. Then, the proposed generalization is the following:

$$
\phi_{i j}^{-2}=\left\{\begin{aligned}
-1 & \text { if } x_{i}=x_{j}=0 \\
1 & \text { if } x_{i}=x_{j}=1 \\
0 & \text { if } x_{i} \neq x_{j}
\end{aligned}\right.
$$


Table 2: Equivalence classes for the subset sum problem with function $\phi_{12}^{-4}$.

\begin{tabular}{|c|c|c|c|c|c|}
\hline \multicolumn{2}{|c|}{$n=2$} & \multicolumn{2}{|c|}{$n=3$} & \multicolumn{2}{|c|}{$n=4$} \\
\hline [00] & [01] & [000] & [001] & [0000] & [0001] \\
\hline 00 & 01 & 000 & 001 & 0000 & 0001 \\
\hline \multirow[t]{7}{*}{11} & 10 & 011 & 010 & 0011 & 0010 \\
\hline & & 100 & 101 & 0100 & 0101 \\
\hline & & 111 & 110 & 0111 & 0110 \\
\hline & & & & 1000 & 1001 \\
\hline & & & & 1011 & 1010 \\
\hline & & & & 1100 & 1101 \\
\hline & & & & 1111 & 1110 \\
\hline
\end{tabular}

Let us now analyze $\phi_{12}^{-4}$ (Figure 5). In Table 2 we show the equivalence classes for this function. The analysis suggests that $\phi_{12}^{-4}=1$ if $x_{1}=x_{2}$ and $\phi_{12}^{-4}=-1$ if $x_{1} \neq x_{2}$. The proposed generalization is the following:

$$
\phi_{i j}^{-4}=\left\{\begin{array}{rl}
1 & \text { if } x_{i}=x_{j} \\
-1 & \text { if } x_{i} \neq x_{j}
\end{array} .\right.
$$

The final proposal of this step can be summarized as follows:

1. The function $\varphi_{i i}$ is an elementary landscape with $\lambda=-2$

2. The function $\varphi_{i j}$ with $i \neq j$ is the weighted sum of two elementary landscapes defined in Equations (35) and (36) with eigenvalues $\lambda=-2$ and $\lambda=-4$, respectively (up to an additive constant).

In the next, and final step, we check the proposal.

\subsection{Step 5: Check the Landscape Decomposition in the General Case}

In this final step we check the functions proposed in the previous step as elementary components of the basic functions. The check consists in a formal proof of the elementariness of the proposed functions or a counterexample showing that they are not elementary. In the case of the formal proof, a relevant result that can be useful is that of Proposition 2. If all the proposed functions are elementary, then we need a final check to complete the landscape decomposition. We need to prove that the weighted sum of the elementary components is the actual basic function. If any of the checks fail, then we can go to Step 4 and try a different proposal.

The reader should note that the previous four steps were required to provide an elementary decomposition proposal for the problem at hand. But we have no proof up to the moment that the decomposition is correct for an arbitrary instance of the problem. In this step, we provide this proof. It is also important to highlight that even though the elementary functions proposed in Step 4 were a result of an inductive reasoning over some small instances of the problem, the result we get in this fifth step is completely general, and can be applied to any instance of any size of the problem. Thus, we should end the fifth step (and the methodology) with a theorem and the proof of that theorem is the operations we do to check the decomposition. 


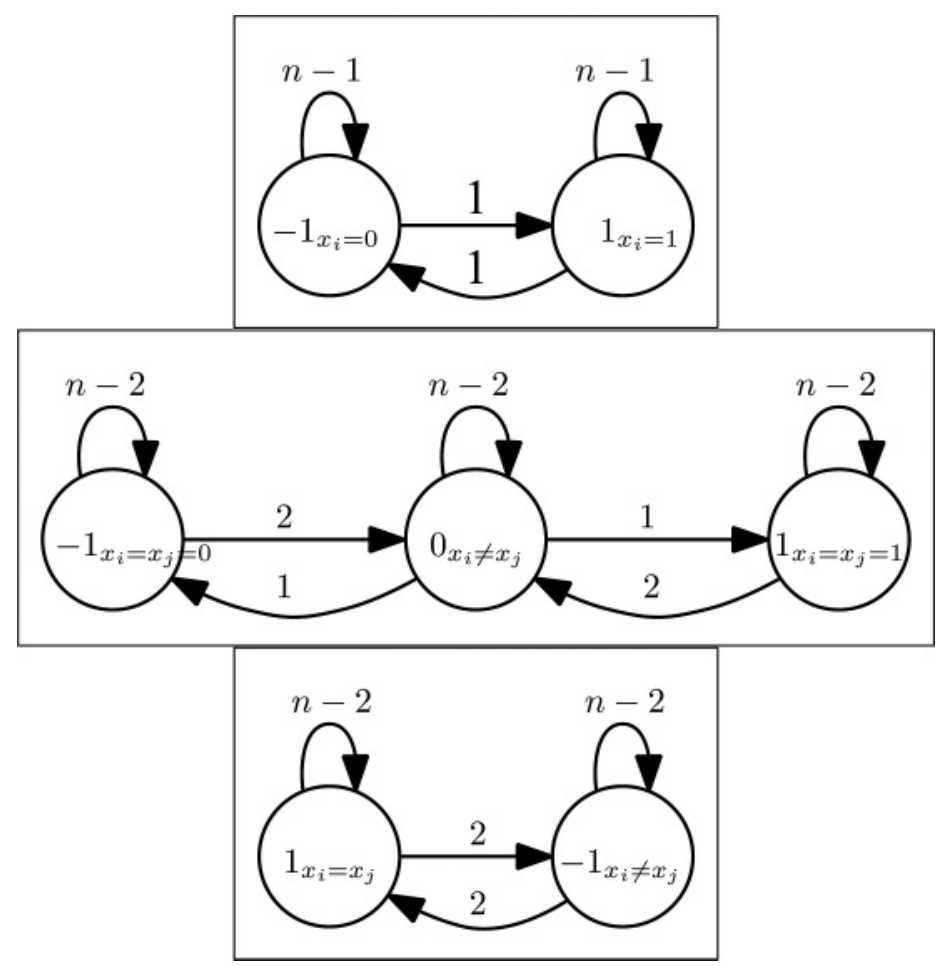

Figure 6: Graphs $G / \sim_{\phi_{i i}^{-2}}, G / \sim_{\phi_{i j}^{-2}}$, and $G / \sim_{\phi_{i j}^{-4}}$.

Let us focus on our example. We start by showing that $\phi_{i i}^{-2}, \phi_{i j}^{-2}$, and $\phi_{i j}^{-4}$ are elementary landscapes with the help of Proposition 2. We will again use the reduced graphs $G / \sim_{\phi}$. However, instead of using the graphs for the particular instances $n=2,3,4$, we use a graph for the general function (arbitrary $n$ ). The general graphs for $\phi_{i i}^{-2}, \phi_{i j}^{-2}$ and $\phi_{i j}^{-4}$ are shown in Figure 6. We can observe that the graphs in Figures 3, 4, and 5 are particular cases of the ones in Figure 6.

Let us prove that the graphs of Figure 6 are the reduced graphs for the corresponding functions. In these graphs, the set of solutions are indicated as predicates in the nodes (the predicates used in the branches of the functions). We must take into account that, by the definition of reduced graph, two nodes of the original graph are in the same equivalence class (node in the reduced graph) if (1) they have the same function value; and (2) all their neighbors are equivalent. In the graphs of Figure 6, all the solutions in each node have the same function value, since the nodes have been defined after the predicates in the branches of the function definition. Then, the first condition is satisfied. In order to check the second condition, we take an arbitrary solution of each node (tentative equivalence class) and we analyze the solution to count how many neighbors the solution has in the other nodes. For example, in graph $G / \sim_{\phi_{i j}^{-2}}$, all the solutions of the node $x_{i} \neq x_{j}$ have one neighbor in the node $x_{j}=x_{i}=1$, which can be obtained by flipping the $x_{j}$ or $x_{i}$ bit which is 0 ; all the solutions also have one neighbor in the node $x_{j}=x_{i}=0$, and the remaining neighbors are in the node $x_{i} \neq x_{j}$. We can carefully analyze in this way the other two nodes of graph $G / \sim_{\phi_{i j}^{-2}}$. If we observe that 
for all solutions in the same node the number of neighbors in the different nodes is the same, then we have a reduced graph. Otherwise, the node is not an equivalence class and we should divide the node into several, each having the same number of neighbors in the same nodes. In the case of the graphs of Figure 6 the reader can note that this last case does not happen and the solutions in the same node are equivalent, thus, they are reduced graphs.

With the help of the graphs we can compute the average value of the functions in the neighborhood of any given solution $x, \operatorname{avg}\{f(y)\}_{y \in N(x)}$ and, thus, we can check if there is a linear relationship between the average and the value of the function in $x$.

Let us consider $\phi_{i i}^{-2}$. For any given solution $x$, it has one neighbor with the opposite value $-\phi_{i i}^{-2}(x)$ and $n-1$ neighbors with the same value $\phi_{i i}^{-2}(x)$. Then, the average can be written as:

$$
\underset{y \in N(x)}{\operatorname{avg}\left\{\phi_{i i}^{-2}(y)\right\}}=\frac{1}{n}\left((n-1) \phi_{i i}^{-2}(x)-\phi_{i i}^{-2}(x)\right)=(1-2 / n) \phi_{i i}^{-2}(x)
$$

and according to Proposition 2 the function is elementary. Furthermore, according to the wave equation, the eigenvalue is $\lambda=-2$, as we conjectured in Step 4 .

We proceed in the same way with $\phi_{i j}^{-2}$. For this function we need to distinguish three cases. They are the following ones:

- Case $\phi_{i j}^{-2}(x)=-1$ : there are two neighbors with $\phi_{i j}^{-2}(y)=0$ and $n-2$ with $\phi_{i j}^{-2}(y)=$ -1 . The average is:

$$
\underset{y \in N(x)}{\operatorname{avg}}\left\{\phi_{i j}^{-2}(y)\right\}=\frac{2-n}{n} .
$$

- Case $\phi_{i j}^{-2}(x)=0$ : there is one neighbor with $\phi_{i j}^{-2}(y)=1$ and another one with $\phi_{i j}^{-2}(y)=-1$. The remaining $n-2$ neighbors have $\phi_{i j}^{-2}(y)=0$. The average is:

$$
\underset{y \in N(x)}{\operatorname{avg}\left\{\phi_{i j}^{-2}(y)\right\}}=0
$$

- Case $\phi_{i j}^{-2}(x)=1$ : there are two neighbors with $\phi_{i j}^{-2}(y)=0$ and $n-2$ with $\phi_{i j}^{-2}(y)=1$. The average is:

$$
\underset{y \in N(x)}{\operatorname{avg}\left\{\phi_{i j}^{-2}(y)\right\}}=\frac{n-2}{n} .
$$

In order for Proposition 2 to be true in this case, there must exist two constants $\alpha$ and $\beta$ such that the following expression holds:

$$
\underset{y \in N(x)}{\operatorname{avg}\left\{\phi_{i j}^{-2}(y)\right\}(x)}=\left(\begin{array}{c}
(2-n) / n \\
0 \\
(n-2) / n
\end{array}\right)=\left(\begin{array}{cc}
-1 & 1 \\
0 & 1 \\
1 & 1
\end{array}\right)\left(\begin{array}{l}
\alpha \\
\beta
\end{array}\right) .
$$

The previous equation holds for $\alpha=1-2 / n$ and $\beta=0$. This confirms that $\phi_{i j}^{-2}$ is an elementary landscape with eigenvalue $\lambda=-2$ (it is a proof). 
Table 3: The basic function $\varphi_{i j}$ and their elementary components $\phi_{i j}^{-2}$ and $\phi_{i j}^{-4}$.

\begin{tabular}{lrcr}
\hline Condition & $\phi_{i j}^{-2}$ & $\phi_{i j}^{-4}$ & $\varphi_{i j}$ \\
\hline$x_{i}=x_{j}=0$ & -1 & 1 & 0 \\
$x_{i}=x_{j}=1$ & 1 & 1 & 1 \\
$x_{i} \neq x_{j}$ & 0 & 0 & 0 \\
\hline
\end{tabular}

Now we consider $\phi_{i j}^{-4}$. For any given solution $x$, it has two neighbors with the opposite value $-\phi_{i j}^{-4}(x)$ and $n-2$ neighbors with the same value $\phi_{i j}(x)$. Then, the average can be written as:

$$
\underset{y \in N(x)}{\operatorname{avg}\left\{\phi_{i j}^{-4}(y)\right\}}=\frac{1}{n}\left((n-2) \phi_{i j}^{-4}(x)-2 \phi_{i j}^{-4}(x)\right)=(1-4 / n) \phi_{i j}(x)
$$

and according to Proposition 2 the function is elementary. Furthermore, according to the wave equation, the eigenvalue is $\lambda=-4$, as we conjectured in Step 4 .

We have proven that functions $\phi_{i i}^{-2}, \phi_{i j}^{-2}$, and $\phi_{i j}^{-4}$ are elementary. To complete this step we need to check if $\varphi_{i i}=\alpha_{1} \phi_{i i}^{-2}+\beta_{1}$ for some $\alpha_{1}$ and $\beta_{1}$ and if $\varphi_{i j}=\alpha_{2} \phi_{i j}^{-2}+\beta_{2} \phi_{i j}^{-4}+\gamma_{2}$ for some constants $\alpha_{2}, \beta_{2}$, and $\gamma_{2}$ when $i \neq j$.

In the case of $\varphi_{i i}$, the basic function is easy, since it is not difficult to see that $\varphi_{i i}=\frac{1}{2}\left(\phi_{i i}^{-2}+1\right)$. In fact, we could have proven that $\varphi_{i i}$ is an elementary landscape instead of proving the elementariness of $\phi_{i i}^{-2}$.

For $\varphi_{i j}$ we show the values of the three functions for the different conditions in Table 3.

In order to obtain the values of $\alpha_{2}, \beta_{2}$, and $\gamma_{2}$ (if they exist) we solve the following linear equation system:

$$
\left(\begin{array}{l}
0 \\
1 \\
0
\end{array}\right)=\left(\begin{array}{ccc}
-1 & 1 & 1 \\
1 & 1 & 1 \\
0 & 0 & 1
\end{array}\right)\left(\begin{array}{l}
\alpha_{2} \\
\beta_{2} \\
\gamma_{2}
\end{array}\right)
$$

The solution to the previous system is $\alpha_{2}=\beta_{2}=1 / 2$ and $\gamma_{2}=0$. Then, we can write $\varphi_{i j}$ as:

$$
\varphi_{i j}=\frac{1}{2}\left(\phi_{i j}^{-2}+\phi_{i j}^{-4}\right)
$$

which proves that $\varphi_{i j}$ is the sum of two elementary landscapes with eigenvalues $\lambda=-2$ and $\lambda=-4$. 
Now we can use Equation (16) to write the landscape decomposition of $f$.

$$
\begin{aligned}
f & =\sum_{i, j=1}^{n} s_{i} s_{j} \varphi_{i j}-2 C \sum_{i=1}^{n} s_{i} \varphi_{i i}+C^{2}=\sum_{i=1}^{n} s_{i}\left(s_{i}-2 C\right) \varphi_{i i}+\sum_{\substack{i, j=1 \\
i \neq j}}^{n} s_{i} s_{j} \varphi_{i j}+C^{2} \\
& =\sum_{i=1}^{n} s_{i}\left(s_{i}-2 C\right) \varphi_{i i}+\frac{1}{2} \sum_{\substack{i, j=1 \\
i \neq j}}^{n} s_{i} s_{j}\left(\phi_{i j}^{-2}+\phi_{i j}^{-4}\right)+C^{2} \\
& =\sum_{i=1}^{n} s_{i}\left(s_{i}-2 C\right) \varphi_{i i}+\frac{1}{2} \sum_{\substack{i, j=1 \\
i \neq j}}^{n} s_{i} s_{j} \phi_{i j}^{-2}+\frac{1}{2} \sum_{\substack{i, j=1 \\
i \neq j}}^{n} s_{i} s_{j} \phi_{i j}^{-4}+C^{2} \\
& =f_{-2}+f_{-4}
\end{aligned}
$$

where $f_{-2}$ and $f_{-4}$ are the elementary components of $f$ with eigenvalues $\lambda=-2$ and $\lambda=-4$, and are defined by:

$$
\begin{aligned}
f_{-2} & =\sum_{i=1}^{n} s_{i}\left(s_{i}-2 C\right) \varphi_{i i}+\frac{1}{2} \sum_{\substack{i, j=1 \\
i \neq j}}^{n} s_{i} s_{j} \phi_{i j}^{-2} \\
f_{-4} & =\frac{1}{2} \sum_{\substack{i, j=1 \\
i \neq j}}^{n} s_{i} s_{j} \phi_{i j}^{-4}+C^{2} .
\end{aligned}
$$

At this point we can present the following.

THEOREM 4: In the flip neighborhood, the objective function of the subset sum problem in Equation (15) can be decomposed as the sum of at most two elementary landscapes with eigenvalues $\lambda=-2$ and $\lambda=-4$. The definition of these elementary components are those of Equations (46) and (47).

ProOF: We have already presented the proof before the statement. All the text and formulas from Equations (37) to (47) are part of this proof.

We should note here that the result of the previous theorem is not restricted to some particular instances of the subset sum problem. In fact, it is valid for any instance of the problem in spite of the fact that we used small instances in the process. Thus, it should be clear that the small instances are only used as a help to find an appropriate elementary decomposition, but the result of the methodology is a completely general decomposition.

There exists a different approach to find the landscape decomposition of the subset sum problem that is easier than the proposed methodology in this particular case, namely: we would have analyzed the problem using the Walsh functions. Thanks to these functions, the binary representation together with the flip neighborhood is a wellknown configuration space from the point of view of landscapes theory. In particular, 
it is known that any function with the form $f(x)=\prod_{j=1}^{k} x_{i_{j}}$ can be decomposed in at most $k$ elementary landscapes, where all the $i_{j}$ holds $1 \leq i_{j} \leq n$ (Rana et al., 1998). Furthermore, the eigenvalues of the elementary landscapes are $-2 p$ for $1 \leq p \leq k$. Since the cost function of the subset sum problem is a quadratic polynomial of the $x_{i}$ variables, it can be decomposed in at most two landscapes with eigenvalues -2 and -4 . The elementary components of the cost function can be obtained by using some properties of the Walsh functions. In the next section, we apply the methodology to a more complex example: the quadratic assignment problem (QAP).

\section{A Complex Example: Quadratic Assignment Problem}

The QAP is an NP-hard combinatorial optimization problem (Garey and Johnson, 1979). This problem class has a considerable importance since some other problems can be formulated as special cases of the QAP. One important example is the traveling salesman problem (TSP). The QAP is not an elementary landscape when the swap neighborhood is considered (Angel and Zissimopoulos, 2000a). The solutions for this problem are permutations, and thus the usual neighborhood is the swap neighborhood. We also know that there exist orthogonal bases of eigenvectors for this configuration space (Stadler, 2002). However, they are based on advanced concepts of group theory, so a specialized mathematical knowledge is required to deal with the Fourier expansion of QAP. In contrast to this, the methodology presented here requires only basic concepts of linear algebra. Part of the following derivation was previously outlined in a conference paper by Chicano et al. (2010); in the current paper, we show in detail the parts of the derivation omitted in the cited work.

\subsection{QAP Formulation}

Let $P$ be a set of $n$ facilities and $L$ a set of $n$ locations. For each pair of locations $i$ and $j$, an arbitrary distance is specified $r_{i j}$ and for each pair of facilities $p$ and $q$, a flow is specified $w_{p q}$. The QAP consists of assigning the facilities of $P$ to the locations in $L$ in such a way that the total cost of the assignment is minimized. Each location can only contain one facility and all the facilities must be assigned to a location. For each pair of facilities, the cost is computed as the product of the flow associated to the facilities and the distance between the locations in which the facilities are placed. The total cost is the sum of all the costs associated to each pair of facilities. One solution to this problem is a bijection between $L$ and $P$, that is, $x: L \rightarrow P$ such that $x$ is bijective. Without loss of generality we can just assume that $P=L=\{1,2, \ldots, n\}$ and that each solution $x$ is a permutation in $S_{n}$, the set permutations of $\{1,2, \ldots, n\}$. The cost function to be minimized can be formally defined as:

$$
f(x)=\sum_{i, j=1}^{n} r_{i j} w_{x(i) x(j)}
$$

The neighborhood $N$ considered here is the swap or two-exchange neighborhood, in which two solutions are neighboring if one can be obtained from the other by a swap (exchange of two elements) in the permutation. Formally, $y \in N(x)$ if and only if there exist two different facilities $i, j \in P$ such that $y(i)=x(j), y(j)=x(i)$ and for all the other facilities $k$ it holds $y(k)=x(k)$. 


\subsection{Step 1: Rewrite the Objective Function}

Let us start rewriting Equation (48). In the case of QAP, the information related to the particular instance is included in the distance matrix $\left(r_{i j}\right)$ and the flow matrix $\left(w_{p q}\right)$. It is not difficult to see that Equation (48) can be written using the following linear combination:

$$
f(x)=\sum_{i, j=1}^{n} \sum_{p, q=1}^{n} r_{i j} w_{p q} \delta_{x(i)}^{p} \delta_{x(j)}^{q}
$$

where we used the Kronecker delta. At this point we can go further and deal with a more general objective function. In Equation (49), the value of the product $r_{i j} w_{p q}$ depends on $i, j, p$, and $q$ in a particular way, but it is not the most general one. Using multilinear algebra concepts, the previous product is a four-rank tensor that has been computed as a tensor product of two two-rank tensors (matrices), which is a special case of a four-rank tensor. In the most general case, we can define a four-rank tensor to replace the product. Let us call the new general four-rank tensor $\psi_{i j p q}$ and let us define the parameterized basic function $\varphi_{(i, j),(p, q)}(x)=\delta_{x(i)}^{p} \delta_{x(j)}^{q}$. Then we can rewrite the fitness function as:

$$
f=\sum_{i, j, p, q=1}^{n} \psi_{i j p q} \varphi_{(i, j),(p, q)}
$$

and we can focus our analysis on the family of basic functions $\varphi_{(i, j),(p, q)}$. Now, the objective function of the QAP is just a particular case of our new objective function $f$, in which $\psi_{i j p q}=r_{i j} w_{p q}$.

Let us identify the equivalence classes in the set of the basic functions. If $i \neq j$ and $p=q$, then $\varphi_{(i, j),(p, q)}=0$, and we can discard these functions. On the other hand, for all the pairs of functions $\varphi_{(i, j),(p, q)}$ and $\varphi_{\left(i^{\prime}, j^{\prime}\right),\left(p^{\prime}, q^{\prime}\right)}$ in which $i \neq j, p \neq q, i^{\prime} \neq j^{\prime}, p^{\prime} \neq q^{\prime}$, we can find an automorphism $\pi$ in the configuration graph $G$ such that $\varphi_{\left(i^{\prime}, j^{\prime}\right),\left(p^{\prime}, q^{\prime}\right)}=$ $\varphi_{(i, j),(p, q)} \circ \pi$. In particular, if $q \neq p^{\prime}, p \neq q^{\prime}, j \neq i^{\prime}$, and $i \neq j^{\prime}$, the automorphism $\pi$ is defined as:

$$
\pi(x)=\left(\begin{array}{ll}
i & i^{\prime}
\end{array}\right) \cdot\left(\begin{array}{ll}
j & j^{\prime}
\end{array}\right) \cdot x \cdot\left(\begin{array}{ll}
p & p^{\prime}
\end{array}\right) \cdot\left(q q^{\prime}\right)
$$

where we used the cycle representation of permutations, the terms with the parentheses are swaps, and the dot operator represents the permutation composition. Then, all the functions $\varphi_{(i, j),(p, q)}$ in which $i \neq j$ (and $p \neq q$ ) are equivalent. We can focus our analysis just on one of them, for example, $\varphi_{(1,2),(1,2)}$.

If $i=j$ and $p \neq q$ we have $\varphi_{(i, j),(p, q)}=0$ and, again, we can discard these functions. The functions $\varphi_{(i, i),(p, p)}$ are not equivalent to any function $\varphi_{(i, j),(p, q)}$ in which $i \neq j$, since the number of solutions with $\varphi=1$ is $(n-1)$ ! in the first case and $(n-2)$ ! in the second case. But are all the $\varphi_{(i, i),(p, p)}$ functions equivalent? The answer is yes, because $\varphi_{\left(i^{\prime}, i^{\prime}\right),\left(p^{\prime}, p^{\prime}\right)}=\varphi_{(i, i),(p, p)} \circ \pi$ with the automorphism $\pi(x)=\left(i i^{\prime}\right) \cdot x \cdot\left(p p^{\prime}\right)$. Finally, we can focus the next steps on the two basic functions $\varphi_{(1,1),(1,1)}$ and $\varphi_{(1,2),(1,2)}$. In order to simplify the notation and when there is no ambiguity, we denote by $\varphi_{1}$ the first function and by $\varphi_{2}$ the second function. 
Table 4: Elementary landscape decomposition of the basic functions $\varphi_{1}$ and $\varphi_{2}$ for $n=2,3,4,5$. We show the number of elementary components, the notation used for them, and their eigenvalue.

\begin{tabular}{ccccc}
\hline Function & $n=2$ & $n=3$ & $n=4$ & $n=5$ \\
\hline$\varphi_{1}$ & $\phi_{12}^{-2}$ & $\phi_{13}^{-3}$ & $\phi_{14}^{-4}$ & $\phi_{15}^{-5}$ \\
$\varphi_{2}$ & $\phi_{22}^{-2}$ & $\phi_{23}^{-3}, \phi_{23}^{-6}$ & $\phi_{24}^{-4}, \phi_{24}^{-6}, \phi_{24}^{-8}$ & $\phi_{25}^{-5}, \phi_{25}^{-8}, \phi_{25}^{-10}$ \\
\hline
\end{tabular}

\subsection{Step 2: Compute $\Delta$ and $\varphi$ for Small Instances}

In the QAP, an instance with $n$ facilities has $|X|=n$ ! solutions. Thus, only a few small instances can be used in order to keep all the computations tractable. In particular, we use the values $n=2,3,4,5$. When $n=5$, the Laplacian matrix is $120 \times 120$ and the computer algebra system requires some minutes to compute the eigensystem. We only show here the Laplacians and the $\vec{\varphi}$ vectors when $n \leq 3$ for illustration purposes.

$$
\begin{aligned}
& \Delta_{2}=\left(\begin{array}{cc}
-1 & 1 \\
1 & -1
\end{array}\right) \\
& \Delta_{3}=\left(\begin{array}{cccccr}
-3 & 1 & 1 & 1 & 0 & 0 \\
1 & -3 & 0 & 0 & 1 & 1 \\
1 & 0 & -3 & 0 & 1 & 1 \\
1 & 0 & 0 & -3 & 1 & 1 \\
0 & 1 & 1 & 1 & -3 & 0 \\
0 & 1 & 1 & 1 & 0 & -3
\end{array}\right) \\
& \vec{\varphi}_{1}=\vec{\varphi}_{2}=(1,0)^{T} \quad \text { for } n=2 \\
& \vec{\varphi}_{1}=(1,0,0,1,0,0)^{T} \quad \vec{\varphi}_{2}=(1,0,0,0,0,0)^{T} \quad \text { for } n=3 .
\end{aligned}
$$

\subsection{Step 3: Compute the Projections of $\varphi$ in the Eigenspaces of $\Delta$}

Using a computer algebra system, we computed the projections of $\varphi_{1}$ and $\varphi_{2}$ into the eigenspaces of $\Delta$. In Table 4 we show the eigenfunctions obtained for each basic function $\varphi_{i}$ and each dimension $n$ using the notation $\phi_{i n}^{\lambda}$, where $\lambda$ is the eigenvalue.

For illustration purposes we only show the projections of $\varphi_{1}$ and $\varphi_{2}$ for $n \leq 3$.

$$
\begin{aligned}
\phi_{12}^{-2} & =\phi_{22}^{-2}=\frac{1}{2}(1,-1)^{T} ; \quad \phi_{13}^{-3}=\frac{1}{3}(2,-1,-1,2,-1,-1)^{T} \\
\phi_{23}^{-3} & =\frac{1}{3}(2,0,0,0,-1,-1)^{T} ; \quad \phi_{23}^{-6}=\frac{1}{6}(1,-1,-1,-1,1,1)^{T} .
\end{aligned}
$$




\subsection{Step 4: Analyze the Projections and Propose Elementary Components}

Once we know the elementary components of the basic functions for the small instances, we need to propose a general formula for the elementary components. First, we multiply the $\phi$ functions by the smaller positive integer that makes integral all the components of the function. Then, we subtract the most common integer number in order to obtain the greatest number of zeros in the function. This step is not necessary, but it is useful for finding a general rule for the $\phi$ functions. We must recall here that this step of the methodology requires, in principle, human intervention and for this reason it is appropriate to highlight noncommon values in the $\phi$ functions. This is what we did with the previous operations.

With the help of Table 4 and the reduced graphs, we can establish a connection between the elementary components in the different instances. For example, according to Table 4 , the basic function $\varphi_{1}$ is an elementary landscape for $n \leq 5$ with eigenvalue $\lambda=-n$. We conjecture that this is also true for $n \geq 6$. Regarding the second basic function $\varphi_{2}$, we can conjecture that it is composed by at most three elementary landscapes for any problem size $n$. We observe in Table 4 that the cases $n=2$ and $n=3$ are special, since $\varphi_{2}$ is elementary in the first case and the sum of two elementary components in the second case. We will return later to these special cases.

If we analyze the eigenvalues of the elementary components, we observe that for each problem instance the smallest one increases linearly with $n$. In particular, the linear relationship is $\lambda=-n$. The same happens with the largest eigenvalue of each instance in which $n \geq 3$, in this case the linear equation is $\lambda=-2 n$. For $n=4$ and $n=5$, a third elementary component appears. Let us suppose that the eigenvalue of this elementary component also increases linearly with $n$, then it should be $\lambda=-2(n-1)$. Now we make the assumption that $\phi_{22}^{-2}, \phi_{23}^{-3}, \phi_{24}^{-4}$, and $\phi_{25}^{-5}$ are instances of a more general function that is an elementary component for any size $n$ of the problem. We further conjecture that $\phi_{23}^{-6}, \phi_{24}^{-8}$, and $\phi_{25}^{-10}$ are instances of a different function that is also an elementary component. Finally, we conjecture that $\phi_{24}^{-6}$ and $\phi_{25}^{-8}$ are instances of a third elementary component. None of these assumptions need to be true (the truth of the assumptions will be studied in the last step of the methodology), we are just proposing general elementary components to be checked in the next step. Moreover, at this point of the methodology, there is no strong argument against the assumption that $\phi_{23}^{-3}$ and $\phi_{24}^{-6}$ are instances of the same elementary component. The check of the fifth step of the methodology will clarify this.

At this point, we conjecture that $\varphi_{2}$ can be decomposed in at most three elementary landscapes with eigenvalues $-n,-2 n$, and $-2(n-1)$. Now we have to propose the expressions for these elementary components in a general instance of size $n$. The reduced graphs will be helpful for this task. For illustration purposes we show in Figure 7 the reduced graphs for $\phi_{24}^{-4}$ and $\phi_{25}^{-5}$. The interested reader can find the remaining reduced graphs in Appendix A. We should find connections between the nodes of the reduced graphs for the same elementary component in different instances. This we do in the following.

Let us focus on the hypothetical elementary component with eigenvalue $-n$, denoted as $\phi_{2 n}^{-n}$. The reduced graphs of $\phi_{24}^{-4}$ and $\phi_{25}^{-5}$ are isomorphic and have five different nodes. This means that the general elementary component most probably will take at most five values. We can examine the solutions in each equivalence class of the graphs in order to search for a connection between the nodes of the two graphs. The nodes with labels -3 and -4 in the reduced graphs of $\phi_{24}^{-4}$ and $\phi_{25}^{-5}$, respectively, could 

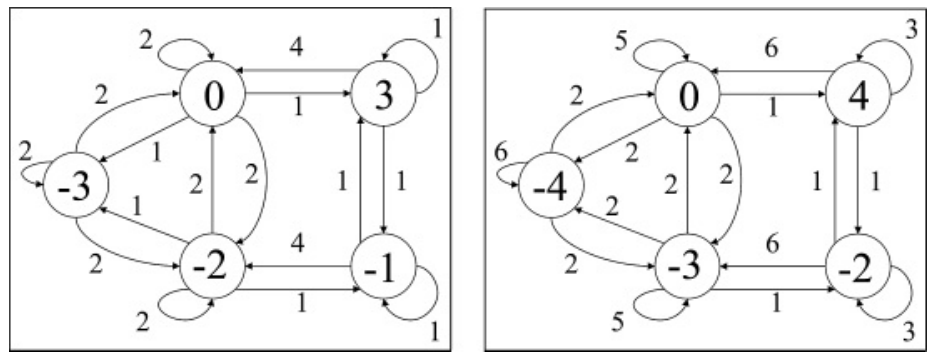

Figure 7: Reduced graphs for $\phi_{24}^{-4}$ (left) and $\phi_{25}^{-5}$ (right).

Table 5: Equivalence classes of nodes labeled with -3 in the reduced graph of $\phi_{24}^{-4}$ and with -4 in the reduced graph of $\phi_{25}^{-5}$.

\begin{tabular}{|c|c|c|c|c|c|}
\hline \multirow{2}{*}{$\frac{\overline{\text { Node }-3 \text { of } \phi_{24}^{-4}}}{[3,4,1,2]}$} & \multicolumn{5}{|c|}{ Node -4 of $\phi_{25}^{-5}$} \\
\hline & {$[3,5,4,2,1]$} & {$[5,4,3,1,2]$} & {$[3,4,1,2,5]$} & {$[5,3,1,2,4]$} & {$[3,4,2,5,1]$} \\
\hline$[3,4,2,1]$ & {$[5,4,1,2,3]$} & {$[5,3,2,4,1]$} & {$[5,4,1,3,2]$} & {$[5,4,2,1,3]$} & {$[4,5,2,3,1]$} \\
\hline$[4,3,1,2]$ & {$[5,3,4,1,2]$} & {$[3,4,1,5,2]$} & {$[5,3,2,1,4]$} & {$[4,3,5,1,2]$} & {$[3,5,4,1,2]$} \\
\hline \multirow[t]{5}{*}[4,3,2,1]{} & {$[4,5,1,3,2]$} & {$[3,5,1,2,4]$} & {$[4,3,2,5,1]$} & {$[4,5,3,2,1]$} & {$[3,4,5,1,2]$} \\
\hline & {$[4,3,1,5,2]$} & {$[3,5,1,4,2]$} & {$[5,4,2,3,1]$} & {$[5,4,3,2,1]$} & {$[3,4,2,1,5]$} \\
\hline & {$[3,5,2,4,1]$} & {$[4,3,2,1,5]$} & {$[4,5,2,1,3]$} & {$[5,3,1,4,2]$} & {$[4,3,1,2,5]$} \\
\hline & {$[3,5,2,1,4]$} & {$[5,3,4,2,1]$} & {$[4,5,3,1,2]$} & {$[3,4,5,2,1]$} & {$[4,3,5,2,1]$} \\
\hline & {$[4,5,1,2,3]$} & & & & \\
\hline
\end{tabular}

represent the same equivalence class in different instances, since these nodes are mapped to each other by any isomorphism between the graphs. After analyzing the solutions contained in the equivalence classes (see Table 5), we discover that in all the solutions of these nodes $x(1) \notin\{1,2\}$ and $x(2) \notin\{1,2\}$. Furthermore, the solutions of these classes are the only ones in which this happens. Thus, we conjecture that the mentioned nodes represent the solutions in which $x(1) \notin\{1,2\}$ and $x(2) \notin\{1,2\}$.

If we analyze node by node all the equivalence classes for graphs $\phi_{24}^{-4}$ and $\phi_{25}^{-5}$, we find a one-to-one correspondence between the nodes that can be described as a particular feature (or predicate) of the solutions belonging to each equivalence class. These five predicates are the following.

- $x(1) \notin\{1,2\} \wedge x(2) \notin\{1,2\}$ : nodes -3 in $\phi_{24}^{-4}$ and -4 in $\phi_{25}^{-5}$. This predicate cannot be true in $\phi_{23}^{-3}$ and $\phi_{22}^{-2}$.

- $x(1)=1 \oplus x(2)=2$ (the $\oplus$ operator denotes the exclusive or): nodes 0 in $\phi_{24}^{-4}$ and $\phi_{25}^{-5}$. In $\phi_{22}^{-2}$ this predicate cannot be true and in $\phi_{23}^{-3}$ the solutions fulfilling this predicate are included in node 0 .

- $x(1)=2 \oplus x(2)=1$ : nodes -1 in $\phi_{23}^{-3},-2$ in $\phi_{24}^{-4}$, and -3 in $\phi_{25}^{-5}$. In $\phi_{22}^{-2}$ this predicate cannot be true.

- $x(1)=1 \wedge x(2)=2$ : nodes 0 in $\phi_{22}^{-2}, 2$ in $\phi_{23}^{-3}, 3$ in $\phi_{24}^{-4}$, and 4 in $\phi_{25}^{-5}$.

- $x(1)=2 \wedge x(2)=1$ : nodes -1 in $\phi_{22}^{-2},-1$ in $\phi_{24}^{-4}$, and -2 in $\phi_{25}^{-5}$. In $\phi_{23}^{-3}$ the solutions fulfilling this predicate are included in node 0 . 
In the previous classification we observe that the labels of the nodes in each equivalence class change in a linear way with respect to $n$. The only exception is that of $\phi_{22}^{-2}$. Then, we take into account this fact to propose a general expression for $\phi_{2 n}^{-n}$ when $n \geq 3$. The proposal is the following:

$$
\phi_{2 n}^{-n}= \begin{cases}n-1 & \text { if } x(i)=p \wedge x(j)=q \\ 3-n & \text { if } x(i)=q \wedge x(j)=p \\ 0 & \text { if } x(i)=p \oplus x(j)=q \\ 2-n & \text { if } x(i)=q \oplus x(j)=p \\ 1-n & \text { if } x(i) \notin\{p, q\} \wedge x(j) \notin\{p, q\}\end{cases}
$$

where we now again use $i \neq j$ instead of 1 and 2 , and we also again introduce the $p \neq q$. Equation (58) is a hypothetical elementary component of the basic function $\varphi_{(i, j),(p, q)}$ where $i \neq j$ and $p \neq q$. We previously saw that $\phi_{22}^{-2}=\phi_{12}^{-2}$ are elementary landscapes. Thus, we can treat $n=2$ as a special case in which the QAP is an elementary landscape due to the elementariness of $\phi_{12}^{-2}$ and $\phi_{22}^{-2}$.

Let us now focus on the hypothetical elementary component with eigenvalue $-2 n$, denoted with $\phi_{2 n}^{-2 n}$. The reduced graphs of $\phi_{24}^{-8}$ and $\phi_{25}^{-10}$ (shown in Appendix A) are isomorphic and have five different nodes. Furthermore, they are isomorphic with $\phi_{24}^{-4}$ and $\phi_{25}^{-5}$. After examining the solutions in each node, we find that the equivalence classes are the same as in the previous function $\phi_{2 n}^{-n}$. We also observe that there is a linear relationship between the values of the nodes and $n$. An analysis of the equivalence classes similar to the one used for the $\phi_{2 n}^{-n}$ functions suggests the following proposal for $\phi_{2 n}^{-2 n}$ when $n \geq 3$ :

$$
\phi_{2 n}^{-2 n}=\left\{\begin{array}{ll}
n-1 & \text { if } x(i)=p \wedge x(j)=q \\
3-n & \text { if } x(i)=q \wedge x(j)=p \\
0 & \text { if } x(i)=p \oplus x(j)=q \\
2 & \text { if } x(i)=q \oplus x(j)=p \\
1 & \text { if } x(i) \notin\{p, q\} \wedge x(j) \notin\{p, q\}
\end{array} .\right.
$$

The previous proposal explains why the hypothetical elementary component $\phi_{2 n}^{-2 n}$ is not present in $\varphi_{2}$ when $n=2$. The reason is that the three last branches of the function definition cannot be true if $n=2$ and for the two first branches the value of $\phi_{22}^{-4}$ is 1 , so the function is a constant function (elementary component with $\lambda=0$ ), and its effect is a change in the average value of $\varphi_{2}$.

Finally, let us focus on the hypothetical elementary component with eigenvalue $-2(n-1)$, denoted as $\phi_{2 n}^{-2(n-1)}$. In this case, the reduced graphs of $\phi_{24}^{-6}$ and $\phi_{25}^{-8}$ are not isomorphic. However, after examining the solutions in each node and analyzing the 
equivalence classes we find the following proposal for $\phi_{2 n}^{-2(n-1)}$ when $n \geq 4$ :

$$
\phi_{2 n}^{-2(n-1)}= \begin{cases}n-3 & \text { if } x(i)=p \wedge x(j)=q \\ n-3 & \text { if } x(i)=q \wedge x(j)=p \\ 0 & \text { if } x(i)=p \oplus x(j)=q \\ 0 & \text { if } x(i)=q \oplus x(j)=p \\ 1 & \text { if } x(i) \notin\{p, q\} \wedge x(j) \notin\{p, q\}\end{cases}
$$

where we used the same branching scheme of Equations (58) and (59) for clarity.

The previous proposal explains why the hypothetical elementary component $\phi_{2 n}^{-2(n-1)}$ is not present in $\varphi_{2}$ when $n=2$, 3. If $n=2$ the three last branches of the function definition cannot be true and for the two first branches the value is -1 , so the function is a constant function. If $n=3$, the last branch cannot be true, and the remaining branches take value 0 , so the function is again a constant function.

At this point we have a proposal for the elementary components of all the basic functions $\varphi_{(i, j),(p, q)}$. Now, in the next step we have to check that the proposed elementary components are really elementary components and we need to compute the value of the weights that these elementary components have in the sum to give the basic functions.

\subsection{Step 5: Check the Landscape Decomposition in the General Case}

In this step, we check the decomposition deduced in the previous step. First, let us focus on the basic functions $\varphi_{(i, i),(p, p)}$. In the previous step, we conjectured that these basic functions are elementary with eigenvalue $\lambda=-n$. Let us study whether this is true with the help of the characterization of elementary landscapes given by Proposition 2 .

In the following, for the sake of clarity we will remove all the parameters from the name of the function when there is no confusion. The function $\varphi$ is elementary if and only if there exist two constants $a$ and $b$ such that the following expression holds for all the solutions:

$$
\underset{y \in N(x)}{\operatorname{avg}\{\varphi(y)\}}=a \varphi(x)+b
$$

In order to reduce the expressions, we multiply the previous expression by the size of the neighborhood, which is $d=\frac{n(n-1)}{2}$. We then obtain:

$$
\sum_{y \in N(x)} \varphi(y)=c \varphi(x)+e
$$

where $c=a d$ and $e=b d$. Next, we compute the exact expression of $\sum_{y \in N(x)} \varphi(y)$ for the two different values that $\varphi$ can take:

- Case $\varphi(x)=1$ (in this case $x(i)=p$ ). From the neighboring solutions, there are $n-1$ with $\varphi(y)=0$ and the remaining neighbors have a value $\varphi(y)=1$. Then we can write:

$$
\sum_{y \in N(x)} \varphi(y)=(d-n+1) .
$$


- Case $\varphi(x)=0$ (in this case $x(i) \neq p$ ). From the neighboring solutions there is only one with $\varphi(y)=1$. The remaining neighbors have a value $\varphi(y)=0$. Then we can write:

$$
\sum_{y \in N(x)} \varphi(y)=1 .
$$

Now we use Equation (61) to obtain the following linear equation system:

$$
\left(\begin{array}{ll}
1 & 1 \\
0 & 1
\end{array}\right)\left(\begin{array}{l}
c \\
e
\end{array}\right)=\left(\begin{array}{c}
d-n+1 \\
1
\end{array}\right) .
$$

The solution of the previous system is $c=d-n$ and $e=1$; so we have $a=1-n / d$ and $b=1 / d$. We conclude that $\varphi_{(i, i),(p, p)}$ is an elementary landscape with $\lambda=-n$.

Now, let us focus on the landscape decomposition of $\varphi_{(i, j),(p, q)}$ for $i \neq j$ and $p \neq q$. Our conjecture in this case is that this function is a weighted sum of the three hypothetical elementary components defined in Equations (58), (59), and (60). We first have to prove that the hypothetical elementary components are really elementary components in the general case. We will exploit the similar structure of the three functions to prove their elementariness at the same time. With this aim, let us define the following parameterized function:

$$
\phi_{(i, j),(p, q)}^{\alpha, \beta, \gamma, \varepsilon, \zeta}(x)= \begin{cases}\alpha & \text { if } x(i)=p \wedge x(j)=q \\ \beta & \text { if } x(i)=q \wedge x(j)=p \\ \gamma & \text { if } x(i)=p \oplus x(j)=q \\ \varepsilon & \text { if } x(i)=q \oplus x(j)=p \\ \zeta & \text { if } x(i) \notin\{p, q\} \wedge x(j) \notin\{p, q\}\end{cases}
$$

where $1 \leq i, j, p, q \leq n$ are integer values with $i \neq j$ and $p \neq q$ and $\alpha, \beta, \gamma, \varepsilon, \zeta \in \mathbb{R}$. In Figure 8 we show the reduced graph of this parameterized function. Again, using the concept of equivalence of solutions, we can prove that the graph in this figure is the reduced graph for $\phi_{(i, j),(p, q)}^{\alpha, \beta, \gamma, \xi}$. For example, in node $\alpha$ the solutions satisfy the condition $x(i)=p \wedge x(j)=q$. Such solutions have exactly one neighbor in node $\beta$ (obtained by swapping positions $i$ and $j$ ), 2(n-2) solutions in node $\gamma$ (swapping either $i$ or $j$ with a third position $k$ ), and the remaining solutions in $\alpha$ (when positions $i$ and $j$ are unaffected). This analysis can be extended to the remaining nodes and we finally conclude that it is a reduced graph.

We should note, however, that depending on the values of the parameters $\alpha, \beta, \gamma$, $\varepsilon$, and $\zeta$ it would be possible to collapse some nodes in the graph. For example, if $\alpha=\beta$ and $\gamma=\varepsilon$, we would collapse the corresponding nodes obtaining a three-node reduced graph. Thus, the graph of Figure 8 is not always the reduced graph. Fortunately, this is not important, because we do not need the reduced graph for the proof, but a graph small enough having different equivalence classes in different nodes. It does not matter if one equivalence class is scattered in different nodes.

Again, for the sake of clarity we will remove all the parameters from the name of the function when there is no confusion. The function $\phi$ is elementary if and only if 


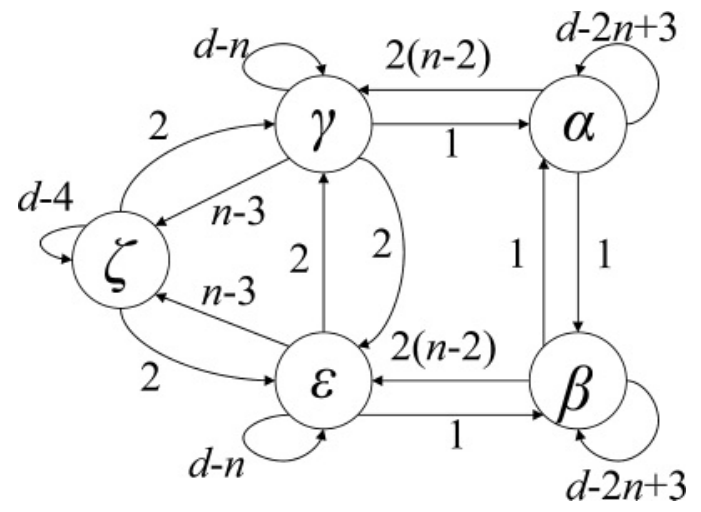

Figure 8: Reduced graph for $\phi_{(i, j),(p, q)}^{\alpha, \beta, \gamma, \varepsilon, \zeta}$.

there exist two constants $a$ and $b$ such that the following expression holds for all the solutions:

$$
\underset{y \in N(x)}{\operatorname{avg}\{\phi(y)\}}=a \phi(x)+b .
$$

In order to reduce the expressions we multiply the previous expression by the size of the neighborhood, which is $d=\frac{n(n-1)}{2}$. We then obtain:

$$
\sum_{y \in N(x)} \phi(y)=c \phi(x)+e
$$

where $c=a d$ and $e=b d$.

Next, we compute the exact expression of $\sum_{y \in N(x)} \phi(y)$ for the five different values that $\phi$ can take:

- Case $\phi(x)=\alpha$. In this case $x(i)=p$ and $x(j)=q$. From the neighboring solutions there is one with $\phi(y)=\beta$ and $2(n-2)$ solutions with $\phi(y)=\gamma$. The remaining neighbors have a value $\phi(y)=\alpha$. Then we can write:

$$
\sum_{y \in N(x)} \phi(y)=\beta+2(n-2) \gamma+(d-2 n+3) \alpha .
$$

- Case $\phi(x)=\beta$. In this case $x(i)=q$ and $x(j)=p$. From the neighboring solutions there is one with $\phi(y)=\alpha$ and $2(n-2)$ solutions with $\phi(y)=\varepsilon$. The remaining neighbors have a value $\phi(y)=\beta$. Then we can write:

$$
\sum_{y \in N(x)} \phi(y)=\alpha+2(n-2) \varepsilon+(d-2 n+3) \beta .
$$

- Case $\phi(x)=\gamma$. In this case $x(i)=p$ or $x(j)=q$, but not both. From the neighboring solutions there is one with $\phi(y)=\alpha$, two neighbors with $\phi(y)=\varepsilon$, and $n-3$ 
neighbors with $\phi(y)=\zeta$. The remaining neighbors have a value $\phi(y)=\gamma$. Then we can write:

$$
\sum_{y \in N(x)} \phi(y)=\alpha+2 \varepsilon+(n-3) \zeta+(d-n) \gamma
$$

- Case $\phi(x)=\varepsilon$. In this case, $x(i)=q$ or $x(j)=p$, but not both. From the neighboring solutions there is one with $\phi(y)=\beta$, two neighbors with $\phi(y)=\gamma$, and $n-3$ neighbors with $\phi(y)=\zeta$. The remaining neighbors have a value $\phi(y)=\varepsilon$. Then we can write:

$$
\sum_{y \in N(x)} \phi(y)=\beta+2 \gamma+(n-3) \zeta+(d-n) \varepsilon .
$$

- Case $\phi(x)=\zeta$. In this case, $x(i) \notin\{p, q\}$ and $x(j) \notin\{p, q\}$. From the neighboring solutions there are two with $\phi(y)=\gamma$ and two neighbors with $\phi(y)=\varepsilon$. The remaining neighbors have a value $\phi(y)=\zeta$. Then we can write:

$$
\sum_{y \in N(x)} \phi(y)=2 \gamma+2 \varepsilon+(d-4) \zeta
$$

We use Equation (63) to obtain the following system of linear equations.

$$
\left(\begin{array}{ll}
\alpha & 1 \\
\beta & 1 \\
\gamma & 1 \\
\varepsilon & 1 \\
\zeta & 1
\end{array}\right)\left(\begin{array}{l}
c \\
e
\end{array}\right)=\left(\begin{array}{r}
\beta+2(n-2) \gamma+(d-2 n+3) \alpha \\
\alpha+2(n-2) \varepsilon+(d-2 n+3) \beta \\
\alpha+2 \varepsilon+(n-3) \zeta+(d-n) \gamma \\
\beta+2 \gamma+(n-3) \zeta+(d-n) \varepsilon \\
2 \gamma+2 \varepsilon+(d-4) \zeta
\end{array}\right) .
$$

The previous system has five equations and two variables, $c$ and $e$, so it could be unsolvable. However, the system can be solved for some value combinations of $\alpha, \beta, \gamma$, $\varepsilon, \zeta$. In particular, the system can be solved for the value combinations we are interested in, that is:

1. $\alpha=n-1, \beta=3-n, \gamma=0, \varepsilon=2-n, \zeta=1-n$ (function $\phi_{2 n}^{-n}$ )

2. $\alpha=n-1, \beta=3-n, \gamma=0, \varepsilon=2, \zeta=1$ (function $\phi_{2 n}^{-2 n}$ )

3. $\alpha=n-3, \beta=n-3, \gamma=0, \varepsilon=0, \zeta=1$ (function $\phi_{2 n}^{-2(n-1)}$ )

This does not mean that these are the only combinations of parameter values for which the system can be solved. They are just three combinations of special interest for the goal of this section. It should be noted here that the linear system does not depend on the values of $i, j, p$, and $q$. Thus, the solutions to the system are also independent of the values of the mentioned parameters.

Let us study the values of $a, b, c$, and $e$ for the first parameter combination, that is, $\alpha=n-1, \beta=3-n, \gamma=0, \varepsilon=2-n$, and $\zeta=1-n$. The solution of the linear 
system is $c=d-n$ and $e=n(3-n)$, and, thus: $a=1-n / d$ and $b=n(3-n) / d$. Thus, we conclude that $\phi_{2 n}^{-n}$ is an elementary function with $\lambda=-n$.

Let us now focus on the second parameter combination, that is, $\alpha=n-1, \beta=3-n$, $\gamma=0, \varepsilon=2$, and $\zeta=1$. The solution of the linear system is $c=d-2 n$ and $e=2 n$, and, thus: $a=(1-2 n / d)$ and $b=2 n / d$. Thus, we conclude that $\phi_{2 n}^{-2 n}$ is an elementary function with $\lambda=-2 n$.

Finally, let us analyze the third parameter combination, that is, $\alpha=\beta=n-3, \gamma=$ $\varepsilon=0$, and $\zeta=1$. The solution of the linear system is $c=d-2(n-1)$ and $e=2(n-3)$, and, thus: $a=1-2(n-1) / d$ and $b=2(n-3) / d$. Thus, we conclude that $\phi_{2 n}^{-2(n-1)}$ is an elementary function with $\lambda=-2(n-1)$.

With the previous arguments, we have proven that the three proposed functions $\phi_{2 n}^{-n}, \phi_{2 n}^{-2 n}$, and $\phi_{2 n}^{-2(n-1)}$ are elementary components. Now, we need to prove that the basic functions $\varphi(i, j),(p, q)$ with $i \neq j$ and $p \neq q$ can be written as a weighted sum of the previous functions. That is, we have to prove that

$$
\varphi_{(i, j),(p, q)}=\omega_{1} \phi_{2 n,(i, j),(p, q)}^{-n}+\omega_{2} \phi_{2 n,(i, j),(p, q)}^{-2 n}+\omega_{3} \phi_{2 n,(i, j),(p, q)}^{-2(n-1)}+\omega_{4}
$$

for some $\omega_{1}, \omega_{2}, \omega_{3}$, and $\omega_{4}$. Since the previous equation must hold for all $x \in X$, it must also hold for each branch in the definition of the $\phi$ functions and $\varphi$. Then, we can find the values of the weights by solving the following linear equation system.

$$
\left(\begin{array}{cccc}
n-1 & n-1 & n-3 & 1 \\
3-n & 3-n & n-3 & 1 \\
0 & 0 & 0 & 1 \\
2-n & 2 & 0 & 1 \\
1-n & 1 & 1 & 1
\end{array}\right)\left(\begin{array}{l}
\omega_{1} \\
\omega_{2} \\
\omega_{3} \\
\omega_{4}
\end{array}\right)=\left(\begin{array}{l}
1 \\
0 \\
0 \\
0 \\
0
\end{array}\right) .
$$

The solution of the previous system is $\omega_{1}=\frac{1}{n(n-2)}, \omega_{2}=\frac{1}{2 n}, \omega_{3}=\frac{1}{2(n-2)}$, and $\omega_{4}=0$. Then we can write:

$$
\varphi_{(i, j),(p, q)}=\frac{\phi_{2 n,(i, j),(p, q)}^{-n}}{n(n-2)}+\frac{\phi_{2 n,(i, j),(p, q)}^{-2 n}}{2 n}+\frac{\phi_{2 n,(i, j),(p, q)}^{-2(n-1)}}{2(n-2)}
$$

for $i \neq j$ and $p \neq q$.

Since the $\phi$ family of functions are elementary, the $\varphi$ family of functions are a sum of three elementary components. This decomposition of $\varphi$ allows us to write the fitness function $f$ as a decomposition of elementary landscapes in the following way:

$$
\begin{aligned}
& f=\sum_{\substack{i, j, p, q=1 \\
i \neq j \\
p \neq q}}^{n} \psi_{i j p q} \varphi_{(i, j),(p, q)}+\sum_{i, p=1}^{n} \psi_{i i p p} \varphi_{(i, i),(p, p)} \\
& \quad=\sum_{\substack{i, j, p, q=1 \\
i \neq j \\
p \neq q}}^{n} \psi_{i j p q}\left(\frac{\phi_{2 n,(i, j),(p, q)}^{-n}}{n(n-2)}+\frac{\phi_{2 n,(i, j),(p, q)}^{-2 n}}{2 n}+\frac{\phi_{2 n,(i, j),(p, q)}^{-2(n-1)}}{2(n-2)}\right)+\sum_{i, p=1}^{n} \psi_{i i p p} \varphi_{(i, i),(p, p)} . \\
&
\end{aligned}
$$


The elementary components of $f$ are:

$$
\begin{aligned}
f_{-n} & =\frac{1}{n(n-2)} \sum_{\substack{i, j, p, q=1 \\
i \neq j \\
p \neq q}}^{n} \psi_{i j p q} \phi_{2 n,(i, j),(p, q)}^{-n}+\sum_{i, p=1}^{n} \psi_{i i p p} \varphi_{(i, i),(p, p)} \\
f_{-2 n} & =\frac{1}{2 n} \sum_{\substack{i, j, p, q=1 \\
i \neq j \\
p \neq q}}^{n} \psi_{i j p q} \phi_{2 n,(i, j),(p, q)}^{-2 n} \\
f_{-2(n-1)} & =\frac{1}{2(n-2)} \sum_{\substack{i, j, p, q=1 \\
i \neq j \\
p \neq q}}^{n} \psi_{i j p q} \phi_{2 n,(i, j),(p, q)}^{-2(n-1)}
\end{aligned}
$$

where the functions $f_{-n}, f_{-2 n}$, and $f_{-2(n-1)}$ are elementary with eigenvalues $\lambda_{1}=-n$, $\lambda_{2}=-2 n$, and $\lambda_{3}=-2(n-1)$, respectively, because they are a linear combination of elementary functions. Thus, $f$ can be written in a compact form as:

$$
f=f_{-n}+f_{-2 n}+f_{-2(n-1)}
$$

Equations (65) to (67) are valid if $n \geq 3$. If $n=2$, there are only two solutions in the search space and the objective function is elementary with eigenvalue $\lambda=-2$ since it can be written as a linear combination of $\varphi_{(i, i),(p, p)}$ functions. At this point we can present the following.

THEOREM 5: In the swap neighborhood the objective function of the QAP in Equation (48) can be decomposed as the sum of at most three elementary landscapes with eigenvalues $\lambda_{1}=-n$, $\lambda_{2}=-2 n$, and $\lambda_{3}=-2(n-1)$. The definition of these elementary components are those of Equations (65), (66), and (67).

Proof: Again we have presented the proof before the statement. The content of the fifth step of the methodology is the proof.

As stated in the previous theorem, the number of elementary components of QAP cannot be larger than three, but it could be lower. For example, we have observed during the application of the methodology that if $n=2$ the QAP is elementary and if $n=3$ there are at most two elementary components with different eigenvalues. It is also possible that for some particular instances the number of elementary landscapes could be reduced. For example, the symmetric TSP is a particular subclass of the QAP that is elementary, the antisymmetric TSP is elementary, and the general asymmetric TSP is the sum of at most two elementary landscapes (Barnes et al., 2002; Stadler, 1996). 
In Appendix B we show the application of the elementary landscape decomposition of the QAP to compute the autocorrelation function of some instances of the QAPLIB (Burkard et al., 1997). We observe that using the elementary landscape decomposition, the autocorrelation values can be computed much faster than using experimental statistical methods.

\section{Limitations of the Methodology}

The methodology presented in this paper is very useful, but it also has limitations. First, it works under the assumption that the number of elementary components of any instance of the problem is bounded by a small constant. This happens in many combinatorial optimization problems, like the subset sum problem, the TSP, or the QAP. But there also exist some problems in which this assumption is not true, like the maximum satisfiability problem (MAX-SAT) or the NK landscapes. An analysis based on Fourier expansions reveals that the maximum number of elementary components in MAX-SAT is the maximum number of literals appearing in any clause, and the maximum number of elementary components in the NK landscapes is $K+1$ (Sutton et al., 2009). Thus, the maximum number of elementary components in any of these problems depends on the particular instance being solved. If our methodology is applied to these problems, the variable number of elementary components could go unnoticed. At the moment we have no rule to guess how many elementary components a function has and we cannot predict from a problem definition whether it can be easily decomposed using the methodology proposed or not. Finding such rules is a line of future work.

Second, the methodology requires human intervention to propose the elementary components, so it depends on the human skills to identify the equivalence classes between the different reduced graphs of different instances. However, as far as we know no methodology exists for decomposing a combinatorial optimization problem into elementary components that can obviate human intervention. Thus, this limitation is not particular to our methodology but general. A fully automated approach would be preferable, but it would also require complete information of the problem and its representation. Our methodology, however, is based on limited knowledge of the problem: Only a few instances of the problem are analyzed and no information of the internal structure of the solutions is used in the systematic steps. We think that this second limitation could be alleviated by using heuristic algorithms to automate the proposal of general elementary components. It is our experience that most of the elementary components are clear after observing the elementary components of the basic functions for small instances. We could program this experience in the form of heuristic algorithms that could suggest to a researcher the general components of a landscape.

Finally, the success of the methodology depends to a large extent on the growth of the search space with the problem size. The methodology requires explicitly dealing with some particular instances of the problem. Only the smallest ones can be used because the size of the search space increases exponentially with the parameters of the instances. This is a consequence of the explicit representation of the basic functions and, specially, the Laplacian matrix. The computation of the eigensystem of the Laplacian requires a computational effort that increases with the Laplacian size. We think that one solution to this problem could be the symbolic manipulation of the Laplacian and the basic functions. 


\section{Conclusion and Future Work}

In this paper we have made three contributions. First, we have presented new theoretical results on landscapes theory that generalize our understanding of elementary landscapes and their properties. Second, we have developed a methodology based on linear algebra that can potentially be used to find a decomposition of a function into a linear combination of elementary components. This decomposition has practical and theoretical applications. In practice, the decomposition allows one to compute the average value of the objective function in the neighborhood of any solution without evaluating all the solutions in the neighborhood. In theory, the decomposition opens the door to the exact computation of the autocorrelation functions and the autocorrelation length in polynomial time. Finally, we have used the methodology to prove that the subset sum problem is a superposition of two elementary landscapes, and the QAP is a superposition of three elementary landscapes.

As future work we distinguish three different lines of research based on the methodology proposed in this paper. First, we plan to apply the methodology to a large number of combinatorial optimization problems for which the elementary landscape decomposition is not known. This decomposition is valuable itself, since it allows one to compute the autocorrelation coefficient for any instance of the problems and could be the basis for new theoretical studies or new operators for evolutionary algorithms. Second, the topic on how the elementary components of a problem can be used to improve the algorithmic performance is an issue that deserves additional research. Third, it is possible to automate most of the operations of the proposed methodology using specific software tools and computer algebra systems. Moreover, we could group together all these operations and develop a single software tool to support the elementary landscape decomposition of a general landscape. Using this tool the researcher could focus on the main creative parts of the methodology: rewriting the objective function and proposing elementary components. We plan to develop such a tool.

\section{Acknowledgments}

We are grateful for the constructive comments from the anonymous reviewers of this paper. This work has been partially funded by the Spanish Ministry of Science and Innovation and FEDER under contract TIN2008-06491-C04-01 (the $\mathrm{M}^{*}$ project). It has also been partially funded by the Andalusian government under contract P07-TIC03044 (DIRICOM project) and by the Air Force Office of Scientific Research, Air Force Materiel Command, USAF, under grant number FA9550-08-1-0422. The U.S. government is authorized to reproduce and distribute reprints for governmental purposes notwithstanding any copyright notation thereon.

\section{References}

Angel, E., and Zissimopoulos, V. (2000a). On the classification of NP-complete problems in terms of their correlation coefficient. Discrete Applied Mathematics, 99:261-277.

Angel, E., and Zissimopoulos, V. (2000b). On the landscape ruggedness of the quadratic assignment problem. Theoretical Computer Sciences, 263:159-172.

Barnes, J., Dokov, S., Acevedo, R., and Solomon, A. (2002). A note on distance matrices yielding elementary landscapes for the TSP. Journal of Mathematical Chemistry, 31(2):233-235. 
Biyikoglu, T., Leyold, J., and Stadler, P. F. (2007). Laplacian eigenvectors of graphs. Lecture Notes in Mathematics. Berlin: Springer-Verlag.

Burkard, R., Karisch, S., and Rendl, F. (1997). QAPLIB -A quadratic assignment problem library. Journal of Global Optimization, 10:391-403.

Chicano, F., Luque, G., and Alba, E. (2010). Elementary landscape decomposition of the quadratic assignment problem. In Proceedings of GECCO, pp. 1425-1432.

Codenotti, B., and Margara, L. (1992). Local properties of some NP-complete problems. Technical Report TR 92-021, International Computer Science Institute, Berkeley, CA.

García-Pelayo, R., and Stadler, P. (1997). Correlation length, isotropy and meta-stable states. Physica D: Nonlinear Phenomena, 107(2-4):240-254.

Garey, M. R., and Johnson, D. S. (1979). Computers and intractability: A guide to the theory of NP-completeness. New York: W.H. Freeman.

Lu, G., Bahsoon, R., and Yao, X. (2010). Applying elementary landscape analysis to searchbased software engineering. In Proceedings of the 2nd International Symposium on Search Based Software Engineering, pp. 3-8.

Rana, S., Heckendorn, R. B., and Whitley, D. (1998). A tractable walsh analysis of SAT and its implications for genetic algorithms. In Proceedings of AAAI, pp. 392-397.

Reidys, C. M., and Stadler, P. F. (2002). Combinatorial landscapes. SIAM Review, 44(1): $3-54$.

Stadler, P. F. (1995). Toward a theory of landscapes. In R. López-Peña, R. Capovilla, R. GarcíaPelayo, H. Waelbroeck, and F. Zertruche (Eds.), Complex systems and binary networks (pp. 77-163). Berlin: Springer-Verlag.

Stadler, P. F. (1996). Landscapes and their correlation functions. Journal of Mathematical Chemistry, 20:1-45.

Stadler, P. F. (2002). Fitness landscapes. In M. Lassig and A. Valleriani (Eds.), Biological evolution and statistical physics (pp. 183-204). Berlin: Springer.

Sutton, A. M., Howe, A. E., and Whitley, L. D. (2010). Directed plateau search for MAX-k-SAT. Paper presented at The 3rd Annual Symposium on Combinational Search, SoCS.

Sutton, A. M., Whitley, L. D., and Howe, A. E. (2009). A polynomial time computation of the exact correlation structure of k-satisfiability landscapes. In Proceedings of GECCO, pp. 365372.

Weinberger, E. (1990). Correlated and uncorrelated fitness landscapes and how to tell the difference. Biological Cybernetics, 63(5):325-336.

Whitley, D., Sutton, A. M., and Howe, A. E. (2008). Understanding elementary landscapes. In Proceedings of GECCO, pp. 585-592.

Whitley, L. D., and Sutton, A. M. (2009). Partial neighborhoods of elementary landscapes. In Proceedings of GECCO, pp. 381-388.

\section{Appendix A Reduced Graphs for Small Instances of QAP}

In Figures 9 to 12 we show all the reduced graphs for the projections of $\varphi_{1}$ and $\varphi_{2}$ and $2 \leq n \leq 5$. 


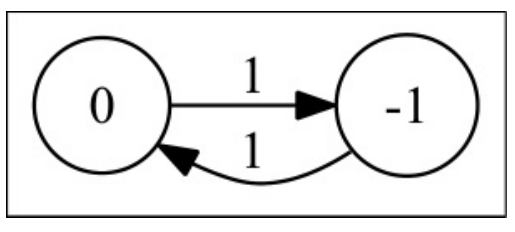

Figure 9: Reduced graphs for the projections of $\varphi_{1}$ and $\varphi_{2}$ when $n=2: \phi_{12}^{-2}=\phi_{22}^{-2}$.
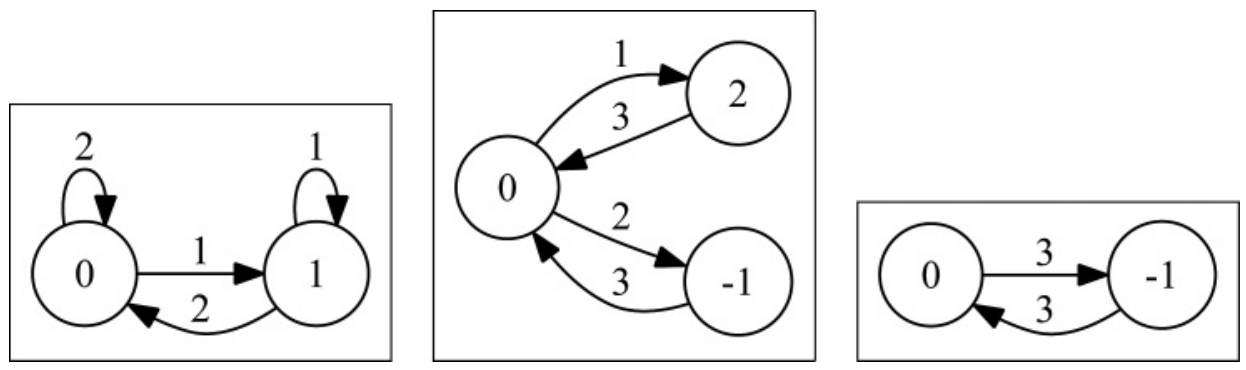

Figure 10: Reduced graphs for the projections of $\varphi_{1}$ and $\varphi_{2}$ when $n=3: \phi_{13}^{-3}$ (left), $\phi_{23}^{-3}$ (center), and $\phi_{23}^{-6}$ (right).
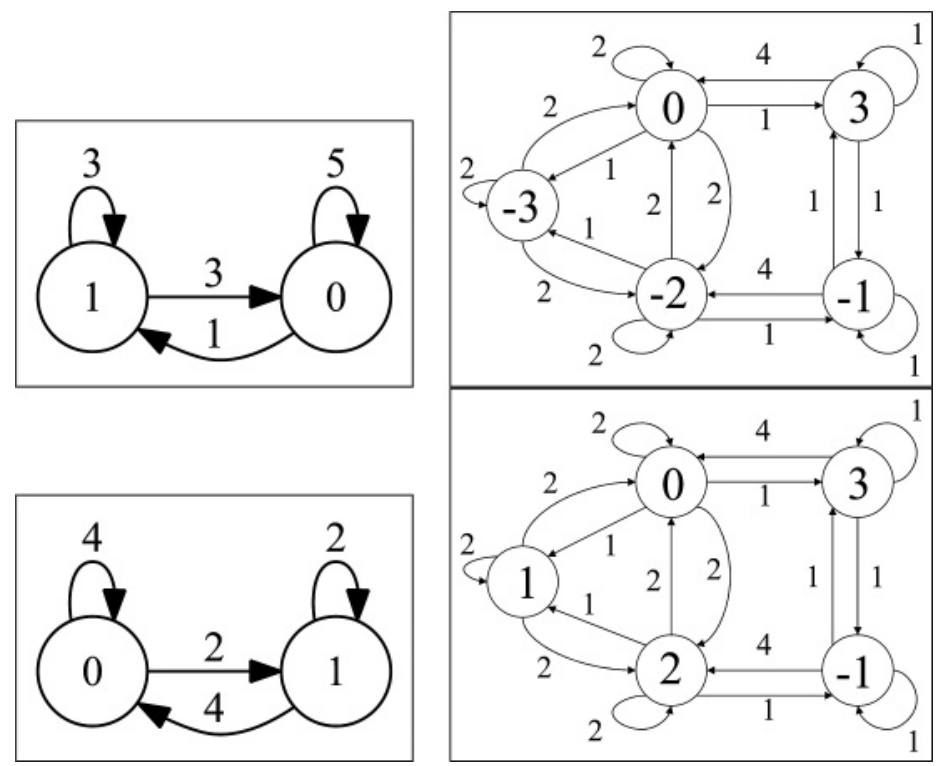

Figure 11: Reduced graphs for the projections of $\varphi_{1}$ and $\varphi_{2}$ when $n=4: \phi_{14}^{-4}$ (top left), $\phi_{24}^{-4}$ (top right), $\phi_{24}^{-6}$ (bottom left), and $\phi_{24}^{-8}$ (bottom right). 

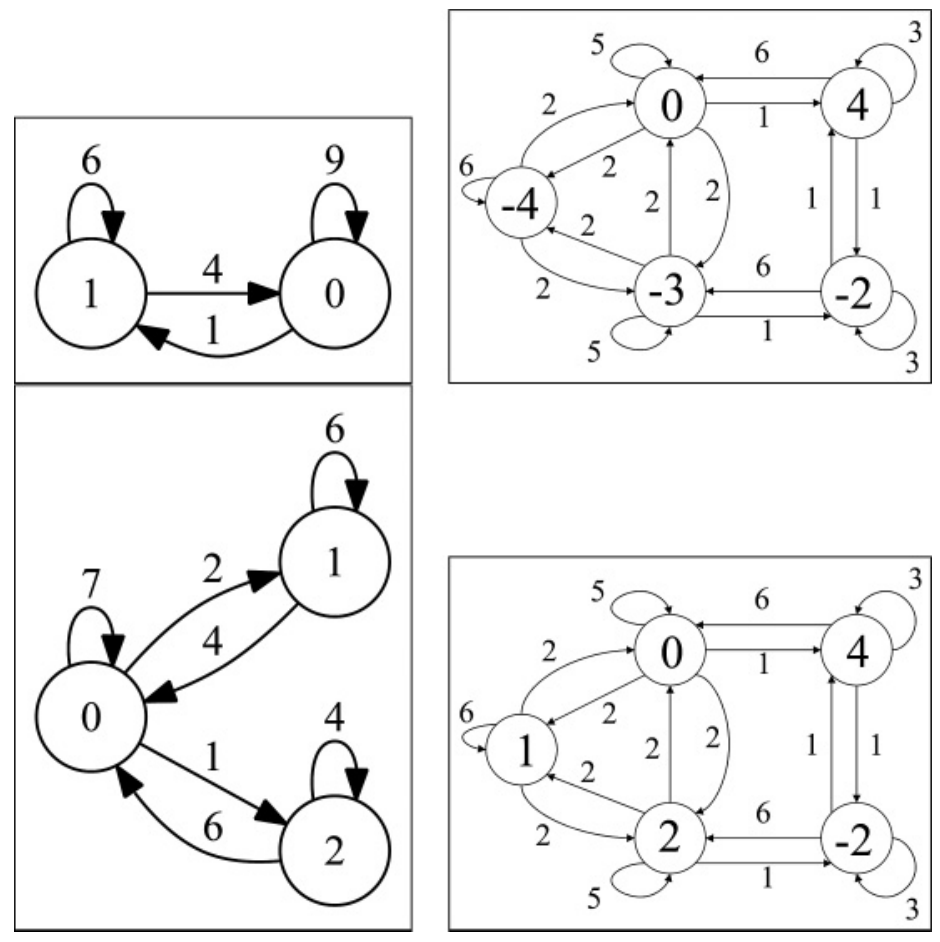

Figure 12: Reduced graphs for the projections of $\varphi_{1}$ and $\varphi_{2}$ when $n=5: \phi_{15}^{-5}$ (top left), $\phi_{25}^{-5}$ (top right), $\phi_{25}^{-8}$ (bottom left), and $\phi_{25}^{-10}$ (bottom right).

\section{Appendix B Autocorrelation of QAP}

Let us consider an infinite random walk $\left\{x_{0}, x_{1}, \ldots\right\}$ on the solution space such that $x_{i+1} \in$ $N\left(x_{i}\right)$. The random walk autocorrelation function $r: \mathbb{N} \rightarrow \mathbb{R}$ is defined as (Weinberger, 1990):

$$
r(s)=\frac{\operatorname{avg}\left\{f\left(x_{t}\right) f\left(x_{t+s}\right)\right\}_{x_{0}, t}-\operatorname{avg}\left\{f\left(x_{t}\right)\right\}_{x_{0}, t}^{2}}{\operatorname{avg}\left\{f\left(x_{t}\right)^{2}\right\}_{x_{0}, t}-\operatorname{avg}\left\{f\left(x_{t}\right)\right\}_{x_{0}, t}^{2}}
$$

where the subindices $x_{0}$ and $t$ indicate that the averages are computed over all the starting solutions $x_{0} \in X$ and along the complete random walk.

The autocorrelation function $r(s)$ can be computed from the actual problem data (instance) using the following expression (Chicano et al., 2010):

$$
r(s)=W_{-n}\left(1-\frac{2}{n-1}\right)^{s}+W_{-2 n}\left(1-\frac{4}{n-1}\right)^{s}+W_{-2(n-1)}\left(1-\frac{4}{n}\right)^{s}
$$

where the coefficients $W_{\lambda}$, called spectral amplitudes, are defined after the elementary components and $f$ as

$$
W_{\lambda}=\frac{\overline{f_{\lambda}^{2}}-{\overline{f_{\lambda}}}^{2}}{\overline{f^{2}}-\bar{f}^{2}} .
$$


Table 6: Experimental (E) and exact (T) values for the autocorrelation function $r(s)$ in six instances of the QAPLIB ( $s$ from 1 to 6 ).

\begin{tabular}{lccccccc}
\hline Instance & & $r(1)$ & $r(2)$ & $r(3)$ & $r(4)$ & $r(5)$ & $r(6)$ \\
\hline tai10a & $\mathrm{E}$ & 0.624255 & 0.393489 & 0.250810 & 0.161890 & 0.106102 & 0.070590 \\
& $\mathrm{~T}$ & 0.624380 & 0.393590 & 0.250903 & 0.162013 & 0.106129 & 0.070617 \\
& $\mathrm{E}$ & 0.749984 & 0.562424 & 0.421759 & 0.316365 & 0.237300 & 0.177939 \\
esc16a & $\mathrm{T}$ & 0.750000 & 0.562500 & 0.421875 & 0.316406 & 0.237305 & 0.177979 \\
& $\mathrm{E}$ & 0.937402 & 0.878700 & 0.823668 & 0.772063 & 0.723672 & 0.678292 \\
esc64a & $\mathrm{T}$ & 0.937500 & 0.878906 & 0.823975 & 0.772476 & 0.724196 & 0.678934 \\
& $\mathrm{E}$ & 0.943369 & 0.890041 & 0.839723 & 0.792267 & 0.747507 & 0.705296 \\
lipa70a & $\mathrm{T}$ & 0.943479 & 0.890170 & 0.839890 & 0.792466 & 0.747735 & 0.705545 \\
& & & & & & & \\
tho150 & $\mathrm{E}$ & 0.975680 & 0.951974 & 0.928863 & 0.906338 & 0.884384 & 0.862981 \\
& $\mathrm{~T}$ & 0.975722 & 0.952060 & 0.928997 & 0.906518 & 0.884607 & 0.863251 \\
& & & & & & & \\
tai256c & $\mathrm{E}$ & 0.984364 & 0.968983 & 0.953843 & 0.938935 & 0.924256 & 0.909805 \\
& $\mathrm{~T}$ & 0.984375 & 0.968994 & 0.953854 & 0.938950 & 0.924279 & 0.909837 \\
\hline
\end{tabular}

We only need to compute two of the three $W_{\lambda}$ values, since $W_{-n}+W_{-2 n}+W_{-2(n-1)}=1$, and we have found an algorithm to compute these values in $O\left(n^{2}\right)$.

In this appendix we check that the autocorrelation measures provided by the elementary landscape decomposition are the same as the ones computed using statistical methods. For this experiment, we have chosen six instances of the QAPLIB (Burkard et al., 1997): two small, two medium, and two large instances. For each instance we have generated one random walk of length 1,000,000 and we have computed the $r(s)$ values for $s \in[0,49]$. This process has been repeated 100 times and we have computed the average value for the 100 independent runs. The results empirically obtained and those theoretically predicted can be found in Table 6 (only for $s \in[1,6]$ ). We can observe a great matching between the empirical and the theoretical value, as expected. The advantage of the theoretical approach is that it is much faster. The experimental results of Table 6 were obtained after 157,783 s of computation (more than $43 \mathrm{hr}$ ). However, the exact values were obtained using Equation (70) in $0.4 \mathrm{~s}$, nearly half a million times faster. 